\title{
Online Sensory Feedback During Active Search Improves Tactile Localization
}

\author{
Xaver Fuchs \\ Bielefeld University
}

\author{
Dirk U. Wulff \\ University of Basel and Max Planck Institute for Human \\ Development, Berlin, Germany
}

\author{
Tobias Heed \\ Bielefeld University
}

\begin{abstract}
Natural motor behavior is usually refined by ongoing sensory input in closed feedback loops. Research has suggested that humans make systematic errors when localizing touch on the skin, and that perceptual body representations underlying these behaviors are distorted. However, experimental procedures usually prevent participants from touching the target limb, interrupting the natural action-perception loop. It is currently unknown how such experimental strategies affect localization and systematic perceptual distortions. Here, participants received a brief touch on their left forearm and, with closed eyes, searched for the target location by moving the right index finger across the left arm. Tactile search significantly reduced the localization error present at touchdown of the searching finger on the target arm. Localization improvement was largely absent when a barrier above the target arm prevented online tactile feedback of the target region. Vision of the arms while reaching to, and searching on, the skin, greatly reduced the localization error at touchdown, but tactile search further improved localization slightly. Thus, both tactile and visual feedback help matching the positions of reaching and target limbs during localization. Yet, even if small, the unique improvement through tactile information confirms the importance of target-related, closed-loop tactile feedback for tactile localization.
\end{abstract}

Public Significance Statement

Reaching toward a touched location on one's body is an elementary behavior. It requires both sensing where touch occurred (perception) and successful reaching (action). Although action and perception are strongly intertwined in many behaviors, tactile research has often neglected this well-known action-perception loop and attempted to study perception in isolation. Here we show that tactile localization critically depends on active, closed-loop behavior: when participants search with their finger for a touched location on their arm, they receive tactile feedback from the arm's skin that allows gradually reducing localization error. Accordingly, many typical experimental tactile localization methods probably underestimate localization ability.

Keywords: tactile localization, perception, active sensing, body representation

Supplemental materials: http://dx.doi.org/10.1037/xhp0000739.supp

This article was published Online First April 23, 2020.

(D) Xaver Fuchs, Biopsychology and Cognitive Neuroscience, Faculty of Psychology and Sports Science, and Excellence Cluster Cognitive Interaction Technology, Bielefeld University; Dirk U. Wulff, Center for Cognitive and Decision Science, University of Basel, and Center for Adaptive Rationality, Max Planck Institute for Human Development, Berlin, Germany; Tobias Heed, Biopsychology and Cognitive Neuroscience, Faculty of Psychology and Sports Science, and Excellence Cluster Cognitive Interaction Technology, Bielefeld University.

All data and code used in this study are available on the website of the Open Science Framework and can be accessed at https://osf.io/ v7hsj.
This research/work was supported by the Emmy Noether Grant of the German Research Foundation (DFG) to Tobias Heed (He 6368/1-1/2/3) and by the DFG-funded Cluster of Excellence Cognitive Interaction Technology (EXC 277) at Bielefeld University. We thank Marta Beauchamp, Joseph Gerges, Diana Kollenda, Nina Held, Vivian Pach, Mathilde Perchermeier, Vitalij Rozkov, Frederick Thiemer, and Lisa Viereck for support with participant recruitment, data acquisition and video processing, Conrad Alting for programming assistance, and Marta Beauchamp for contributing the drawings for Figures 1 and 5 .

Correspondence concerning this article should be addressed to Xaver Fuchs, Biopsychology and Cognitive Neuroscience, Faculty of Psychology and Sports Science, Bielefeld University, Universitätsstr. 25, 33615 Bielefeld, Germany. E-mail:xaver.fuchs@uni-bielefeld.de 
Localizing touch on the skin is a fundamental function of the tactile system. Yet, humans often misjudge tactile location by up to several centimeters, even in seemingly simple tasks such as pointing toward the touched body part (Culver, 1970; Elithorn, Piercy, \& Crosskey, 1953; Geldard, 1985). Different factors may affect localization accuracy. On many body parts, receptor density is surprisingly low, limiting the acuity with which stimulus location on the skin can be detected (Weinstein, 1968). Furthermore, touch occurs on the two-dimensional sheet of the skin and must be combined with posture to derive a location in space, before a goal-directed motor response toward the touched area can be executed; these sensorimotor transformations potentially introduce further error (Heed \& Azañón, 2014; Heed, Buchholz, Engel, \& Röder, 2015; Maij, Medendorp, \& Heed, 2019; Tamè, Azañón, \& Longo, 2019). Lastly, localization error can result from motor error of the acting limb because motor execution is not performed with perfect reliability, as is evident from the observation that reaching endpoint varies across repeated trials even if the movement target is stationary (Harris \& Wolpert, 1998; Trommershäuser, Maloney, \& Landy, 2008).

Researchers have investigated tactile localization with various experimental paradigms, mostly on the upper limbs. Some studies have asked individuals to indicate touch locations on a silhouette shape of the body (Head, 1920; S. Medina, Tamè, \& Longo, 2018) or to assign touch location to a grid drawn on the limb (Weinstein, 1968). Others have required reaching or pointing movements without touching the target limb (Trojan et al., 2010), or with the tactile target hidden under an occluding board (Longo, Mancini, \& Haggard, 2015; J. Medina \& Duckett, 2017) or touch screen (Mueller \& Fiehler, 2014). One important aspect shared by all these methods is that participants, on purpose, do not receive tactile feedback about their localization accuracy from the target region. Thus, all above-mentioned methods are open-loop. In contrast, real-life situations often involve touching one's own skin when localizing the stimulus and, thus, allow closed-loop control, underlining the active nature of seemingly perceptual functions. The open-loop constraint in these experiments is typically based on a purposeful decision aimed to study "pure" tactile localization or "perceptual" body representations and avoid potentially confounding influence of additional tactile information produced during localization. To give one example, open-loop responses of tactile localization on the hand do not map onto the actual dimensions of the hand, suggesting that the brain represents the hand as shorter and wider than it actually is (Longo \& Haggard, 2010; Mancini, Longo, Iannetti, \& Haggard, 2011). Yet, others have criticized these conclusions, arguing that distortions may be due to location estimates being referred to previous estimates, that is, that they may originate from domain-general bias induced by the required behavioral response rather than from true representational distortion (J. Medina \& Duckett, 2017).

Beyond the context of tactile localization, the notion that perception is an active process that encompasses the interplay between sensory and motor processes is a widely accepted idea (Friston, 2010). For instance, tactile object recognition critically depends on manipulating the object with the hands and moving one's fingers across it (Lederman \& Klatzky, 2009). Furthermore, spatial perception in touch can be anchored to the timing of active movement, as demonstrated by neural coding of touch during whisking in rats (Ahissar \& Arieli, 2001), as well as systematic localization errors that arise when humans indicate the location of touch that occurred during arm movements (Maij, Wing, \& Medendorp, 2013).

In vision, saccades into the periphery often slightly miss their target, and are then corrected by secondary saccades, both in the laboratory (Ohl, Brandt, \& Kliegl, 2011; Srimal \& Curtis, 2010) and naturalistic environments (Land, Mennie, \& Rusted, 1999). Note that in this case the visual target falls on the low-resolution periphery of the eye before the first saccade. The spatial location of the target can, therefore, not be precisely determined. The first saccade then moves the visual target into the high-resolution fovea, allowing online assessment of exact target location and, hence, correction of the remaining error.

We hypothesized that a similar closed-loop mechanism may support tactile localization. In particular, initial targeting based on the spatial information derived from the tactile input may be coarse, with online tactile feedback during search providing higher-resolution tactile-spatial information, allowing to refine localization. Imagine scratching your arm: Often, we simply direct our scratching hand grossly toward the itch. Then, we scratch across the itching arm until we find the location the itch originates from.

Notably, two distinct mechanisms may underlie such behavior. A closed-loop account posits that searching movements should be systematically directed toward the target location, that is, the distance between the acting hand and the tactile target should reduce continually. An alternative possibility, however, is that the hand searches randomly, rather than goaldirected, so that success in search would depend on hitting the target location by chance during random-like movements, rather than on a closed-loop sensorimotor control mechanism.

Here, we investigated closed-loop tactile search in a series of four experiments. Experiment 1 established that closed-loop tactile search indeed improves tactile localization performance. Participants received a brief touch on the left forearm and localized the touched position by first guiding the right index finger to the target area, setting down, and then searching by moving the finger across the skin. Participants closed their eyes throughout testing to exclude any influence of online vision. In three follow-up experiments, we addressed potential confounds and alternative explanations of our initial findings. Experiment 2 examined whether the localization improvement through search truly depended on tactile feedback from the target region. To test this question, participants were allowed to search for the target location on a barrier directly above their arm, allowing them to improve their initial aim, but preventing tactile feedback on the target arm. Experiment 3 ensured that our initial results were not an artifact of participants' instructions. Participants localized tactile stimuli through search, either with the instruction to touch down as close to the tactile target as possible, or with no specific instruction as to how to perform the touchdown. Finally, Experiment 4 tested whether the improvement through search is specific to tactile feedback. It tested the alternative hypothesis that search may be improved because some sensory information-but not necessarily touch-improves the estimate of the searching finger's position on the arm. To this end, participants searched with their eyes open. 


\section{Experiment 1}

\section{Method}

The rationale of Experiment 1 was that searching with the index finger on the skin generates online tactile feedback from the target region's skin. The aim was to investigate whether participants improve localization by using this feedback. If localization error, that is, the distance of reported from true stimulus position, is reduced after, as compared to before tactile search, this indicates that participants use tactile feedback around the target for error reduction.

Participants. For Experiment 1, our aim was to assess localization of tactile events in a large and heterogeneous sample, in a setting as natural as possible. For this purpose, we recruited participants at a public science fair in Bielefeld.

We originally recruited 63 adult participants. Five of 63 data sets were excluded due to technical problems during data acquisition. The final sample consisted of 58 participants (aged 14-73, $M=40.5$ years; 36 females, 21 males, in one case sex was not recorded). Fifty participants were right-handed, 5 were bidexterous and 3 were left-handed, according to self-report. The average arm length was $21.9 \mathrm{~cm}$ (range: $18-28 \mathrm{~cm}$ ). Participants gave informed, verbal consent, and did not receive monetary compensation.

General procedures. Participants received brief tactile stimuli on their left dorsal forearm and localized the stimuli using their right hand's index finger. In contrast to previous studies on tactile localization, direct contact with the skin of the target arm was permitted, and localization continued after initial contact with the target arm as a closed-loop, active search; participants were explicitly allowed, and moreover encouraged, to "find" the location of the previously presented touch by moving their index finger across the target arm's skin. Participants closed their eyes throughout the entire trial, including stimulation and localization.

Experimental design. We manipulated two experimental factors. First, target arm posture was either orthogonal (at approxi- mately $90^{\circ}$ ) or parallel to the torso (see Figure 1). Because lowlevel skin maps are thought to be anatomically organized (Calzolari, Azañón, Danvers, Vallar, \& Longo, 2017), common external-spatial error across the two postures would indicate motor error of the pointing hand rather than perceptual localization error on the target arm. We varied this factor blockwise as a withinsubject factor, with the order balanced across participants. Second, target region varied between three different areas along the forearm: a proximal position near the elbow; a middle position halfway between elbow crook and wrist; and a distal position near the wrist. Targets were applied on top of the arm, centered with respect to the mediolateral axis of the arm. Within the three defined regions, we slightly jittered location between trials along the proximodistal axis to avoid adaption and practice effects. Within a given block, each region was touched five times in pseudorandomized order, resulting in five trials per combination of posture and target region.

Tactile stimulation. Tactile stimuli were applied manually by the experimenter using a hand-held, wooden stick (a size 15 paintbrush held upside-down) of approximately $35 \mathrm{~cm}$ length. The stick's tip was blunt, with a diameter of $3 \mathrm{~mm}$, and evoked a light touch sensation. We applied a force that caused a visible dent and slight whitening of the skin in the target region and lasted for approximately $1 \mathrm{~s}$.

Tactile localization. Participants initiated the localization when the tactile stimulus had been released from the skin. Participants reached using their right index finger to the left forearm, touched down, and then moved their finger across the skin until they felt that they had reached the target. Stimulus and finger locations were recorded from above with a camera (see below). For the reaching movement before touchdown on the skin, we instructed participants to point naturally and with moderate speed, in a fluent way, and without interruptions to allow for online corrections of the movement (Desmurget et al., 1999; Kammers, de Vignemont, Verhagen, \& Dijkerman, 2009; Scott, 2004). When participants had completed their search, they kept their finger
A

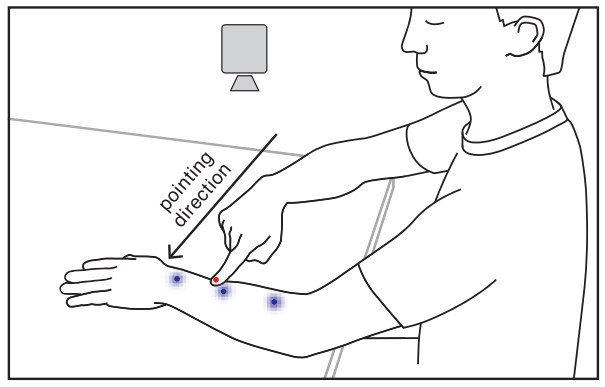

B

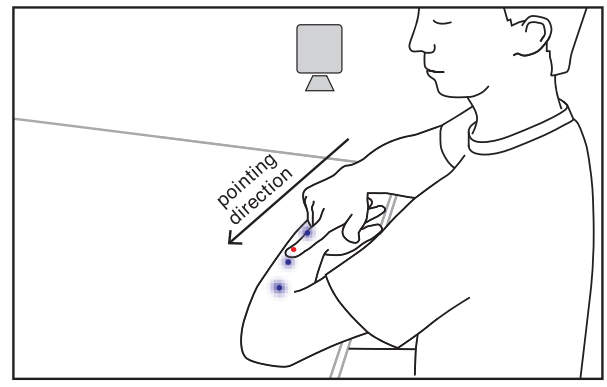

Figure 1. Illustration of the procedures used in Experiment 1. Participants received brief touches, applied with a hand-held wooden stick, in one of three target areas of the dorsal forearm (blue points). Subsequently, they localized the touch with their right hand's index finger. Two posture orientations, straight (A) and angled (B), were used to separate perceptual from motor localization error: The pointing hand approached the target arm from the right (from the participant's view) in both posture orientations. Hence, motor error (e.g., overshoot) would affect different anatomical directions on the target arm for the two postures. See the online article for the color version of this figure. 
stationary in the final position for a few seconds before they returned to the starting position $39 \mathrm{~cm}$ to the right of the target arm. Participants performed several practice trials until they indicated that they had fully understood the procedure.

Acquisition of stimulus positions and search trajectories. We recorded stimulus presentation and participants' finger movement during localization with a digital camera. The camera was mounted above the table and aligned with the table surface to yield undistorted image data. A $0.8 \mathrm{~cm}$ diameter, round, bright orange sticker on the participant's right index finger's nail indicated finger position. We used an Intel RealSense camera (Intel, Santa Clara, CA) at a spatial resolution of $1,280 \times 720$ pixels and a framerate of 30 frames/s that was controlled using custom programming code in PsychoPy (v1.85.2, www.psychopy.org) running within the free Ubuntu operating system (v16.04, www.ubuntu.com) on a Laptop computer (Dell Latitude, Dell, Round Rock, TX).

Estimation of sample size and power. Because the experimental paradigm was new, a priori estimation of sample size was not feasible in Experiment 1. We therefore acquired data from a fairly large sample to ensure high statistical power.

Extraction of target and finger position from image data. We analyzed videos with Matlab (v2016a, www.mathworks.com). First, we marked four anatomical coordinates of the forearm on the image, namely the inside and outside edges of the elbow and of the wrist; marks were identified anew for each individual trial. The four coordinates defined a trapezoid shape that approximately covered the area of the forearm. Second, we identified the frame (i.e., timepoint in the video) in which the tactile stimulus was presented on the skin. We used the frame in which skin indentation was largest and marked stimulus location on the image. Third, we found the frame at which the participant's finger first touched the target arm's skin. Fourth, we found the frame in which the participant lifted off from the target arm. We then extracted the center position of the orange dot that marked index finger position for all frames between touchdown and lift-off. Note, that the time that passed before and after movement on the skin, when the participant's finger stayed idle, is irrelevant for statistical analysis of movement trajectories because we analyzed trajectories with respect to space, independent of time (see Data Processing). For the extraction of the positions from the images, we used a graphical user interface for Matlab, developed in-house, which is available online (see software availability).

Data loss and exclusion. For the 58 participants within the analyzed dataset, at least one trial was missing in 48 (85\%) cases, with an overall trial loss of $15 \%$. The main reasons for data loss were difficulty to extract the finger position from video and occasional data transfer problems of the digital camera. The analysis we present is based on 1,480 trials. In one participant, the information about arm length was missing and the arm length value was substituted by the sample average.

Data preprocessing. All data were analyzed with R (v3.5.1., https://cran.r-project.org/; R Core Team, 2014). First, we spatially aligned all trajectory data to eliminate differences due to posture and individuals' limb size. During data acquisition, we measured the length of the target forearm from the crook of the elbow to the wrist using a measuring tape. For each trial, the arm area was transformed to a mean group template based on the four anatomical coordinates of the elbow and wrist using the "Morpho" pack- age for R (Schlager, 2017). For each single trial, arm area, tactile location, and the search trajectory were transformed into the group template space. Finally, we expressed all coordinates as percent of forearm length (Steenbergen, Buitenweg, Trojan, \& Veltink, 2014), measured as the distance between the middle position between the two elbow coordinates and the middle between the two wrist coordinates of the template. As the outcome variable for statistical analysis, we expressed trajectories relative to target position by subtracting the target position, effectively coding trajectories as continuous localization error. In other words, a value of 0 in our trajectories would indicate that the searching finger was right on the target. For comparison with previous studies, we analyzed trajectories separately in proximodistal and in mediolateral direction. To reduce error into a single, direction-independent measure, we also expressed trajectories as the Euclidian distance between finger and target.

For the analysis of search progression, we "spatialized" trajectories using the R package "mousetrap" (Kieslich, Henninger, Wulff, Haslbeck, \& Schulte-Mecklenbeck, 2019; Wulff, Haslbeck, Kieslich, Henninger, \& Schulte-Mecklenbeck, 2019). We recoded all trajectories into 20 segments (between 21 points) between touchdown and lift-off. Following this step, each trajectory contained the same number of observations, and each segment coded $5 \%$ of the traveled distance. Hence, this analysis step fully reduces trajectories to their spatial features, here segments representing steps of $5 \%$ of the trajectory length. Accordingly, the recoded trajectories are not influenced by the fact that original movement trajectories at constant sample rate usually have many data points at the beginning and at the end due to the lower movement speed (Wulff et al., 2019).

For the analysis of the error remaining after search, we transformed the data into $\mathrm{cm}$ units by multiplying the percentage of arm length values with the participant-specific arm length in $\mathrm{cm}$.

Statistical analysis. We analyzed four properties of localization and evaluated the effect of search on the skin and of the levels of posture and target region.

First, constant error describes the direction-dependent error as distance from the target and represents systematic distortion, or bias, of localization. We computed constant error for initial and final localization (i.e., at touchdown vs. after tactile search on the skin) by averaging the distance to the target in proximodistal (from the elbow to the wrist) and mediolateral direction (from the inside to the outside of the arm) across trials within each participant.

Second, variable error describes the consistency of localization across trials. We analyzed variable error in analogy to constant error, with the only difference that variable error was computed as the standard deviation, rather than the average, across trials. The first two analysis steps can therefore clarify whether perceptual distortion differs before and after search.

Third, absolute error is the absolute value of distance from the target and represents error irrespective of direction.

Fourth, progression of error reduction during search is how the (direction-independent) Euclidian distance to the target changes within search trjaectories. We analyzed how this measure changed in the course of the 20 segments of the search trajectories.

We used linear mixed models, as implemented in the "lme4" (Bates, Mächler, Bolker, \& Walker, 2014) and "lmerTest" (Kuznetsova, Brockhoff, \& Christensen, 2014) packages for R. Linear mixed models account for differences in trial numbers between 
participants and allow for individually sized effects across participants. For the analysis of constant and variable error, we computed a model that included posture (straight and angled), direction (proximodistal and mediolateral), target region (proximal, medial, and distal), and search (initial and final localization) as fixed factors, including all possible interactions. We included participant as a random intercept effect. We also added all random slopes for the main effects and two-way interactions but omitted higher-order interactions in the random effects structure to keep the model identifiable. In lme4 notation, the model was specified as follows: lmer(constError $\sim$ posture ${ }^{*}$ direction ${ }^{*}$ region * search $+(1+\text { (posture }+ \text { direction }+ \text { region }+ \text { search })^{\wedge} 2$ । id $\left.)\right)$, with ${ }^{\wedge}$ as notation of main effects and two-way interactions. This approach of including random slopes for all factors and all twoway interactions was used for all LMMs presented in this article. In case the model resulted in a singular fit or was not identifiable, the two-way interactions in the random effects were dropped, with all random intercepts remaining. In case this model was still singular, we reverted to the random intercept model. For each statistical model, the formula is provided in the online supplementary material. To quantify the magnitude of constant error, we computed estimated marginal means (EMMs) from the model using the "emmeans" package (Lenth, Singmann, Love, Buerkner, $\&$ Herve, 2019). Because trajectories were coded as distance from the target, EMMs significantly different from zero indicate bias (i.e., constant error) in the respective direction. For the absolute error, we computed a model with the same factors, but with unsigned trajectory data as dependent measure. We ran separate models with absolute and variable error, respectively, as dependent measures, but identical factor design.

We analyzed search progression with a similar model that included fixed factors posture and target region. It used error in Euclidian space as dependent measure, rather than separating into proximodistal and mediolateral error, to reduce model complexity. The model expressed progression in the trial as a fixed factor percent moved, which consisted of 20 factor levels resulting from trajectory spatialization. For this factor, we used orthogonal polynomial contrasts of first and second order, that is, a linear and a quadratic term. Furthermore, the model included initial localization error (initially small, initially medium, and initially large) as a fixed factor. This factor was defined by binning trials at percentile 33 and 66 according to the Euclidian distance from the target at touchdown. We ran submodels that included only the data of one factor level to break down significant interactions.

All model formulae are included in the online supplementary material. We considered effects to be statistically significant at $p<$ .05 . We corrected $p$ values for post hoc tests for multiple comparisons using false discovery rate.

\section{Results}

Constant localization error. Constant error expresses the average localization error that is consistent across trials. We observed constant error in the distal, that is, toward the hand, and in the lateral direction, that is, toward the outside of the arm of comparable size (both $1.3 \%$ arm length, both $p<.001$ ). Overall, constant error was of comparable magnitude for initial and final finger location, ruling out a contribution of search on the skin to localization bias, $F(1,93.1)=0.36, p=.548$ and was statistically significant for each posture both before and after search (see Supplemental Table S4 in the online supplementary material). However, although search did not change the general size of the errors, both distal and lateral bias slightly diminished after search in the angled but increased in the straight posture, $F(1,1,030.7)=$ $22.48, p<.001$. Constant error magnitude slightly differed between target regions (Figure 2 and Supplemental Table S2 in the online supplementary material). There was no overall difference between arm postures, $F(1,58.4)=0.03, p=.853$, suggesting that constant error was not a result of movement direction. However, differences between target regions depended on posture $(F(2$, $1060.5)=9.26, p<.001$; see Supplemental Table S1 in the online supplementary material for details). The present distal localization biases are in line with previous reports based on various measurement methods, suggesting distorted body representations (Longo et al., 2015; Mancini, Longo, Iannetti, et al., 2011).

Whereas tactile search did not compensate for perceptual bias, it did reduce the absolute distance from the target, $F(1,1,240.1)=$ $19.18, p<.001$. Error reduction was evident in both distal and lateral direction (Figure 2A vs. 2B; Figure 4A; Supplemental Table S6 in the online supplementary material). Absolute error was larger in proximodistal than in mediolateral direction $(4.5 \%$ vs. $2.9 \%$ arm length, $p<.001$; Figure $2 \mathrm{~A}$ and $2 \mathrm{~B}$ ). Because tactile search reduced localization error in all directions, we conducted further analyses on the Euclidian distance to the target by collapsing error into a single, direction-independent, measure. Tactile search improved final localization in $68-72 \%$ of trials for the different conditions, confirming that error reduction was a general effect and did not, for instance, depend on just a few trials with very large corrections (Figure 3A).

Variable localization error. The reduction of absolute error through tactile search was accompanied by a reduction of variable error, $F(1,1,223)=174.3, p<.001$. Variable error describes the variability in localization performance, that is, the unsystematic error, and is computed as the intrasubject standard deviation across trials. Overall, variable error was larger in proximodistal than in mediolateral direction $(5.3 \%$ vs. $3.3 \%$ arm length, $p<.001$, Figure $2 \mathrm{C}$ and $2 \mathrm{D}$ and Supplemental Table S9 in the online supplementary material). These effects were evident across all conditions, although error was slightly larger in the angled than the straight posture, and it differed between the target regions (Figure 2C and 2D and SSupplemental Tables S8-S10 in the online supplementary material). Similarly, the variance across trials in Euclidian distance was lower for final than for initial localization in 57-81\% of the participants for the different target regions (Figure 3B).

The reduction of variable error, together with the observation that the overall direction of constant error was both unaffected by search and comparable across the two tested arm postures, implies that localization gravitated toward target location and, hence, search reduced localization error for any direction from which the searching finger had started after touching down. This effect of convergence toward the target area from all sides can also be seen in the animated version of Figure 4A, which is available in the online supplementary material (Supplemental Figure S4A).

Progression of error reduction in trajectories. So far, we have established that tactile search reduced absolute and variable localization error. To better characterize the observed error reduction, we analyzed how it developed across the search trajectory and whether it depended on the magnitude of the initial localization 


\section{A Initial constant error}

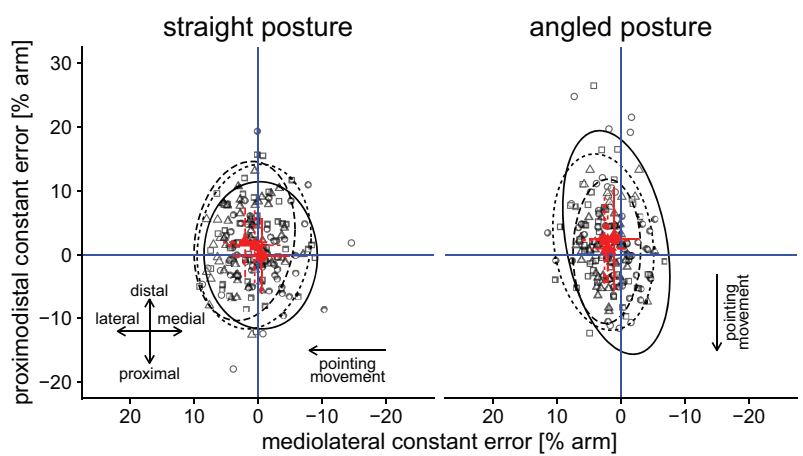

\section{Initial variable error}

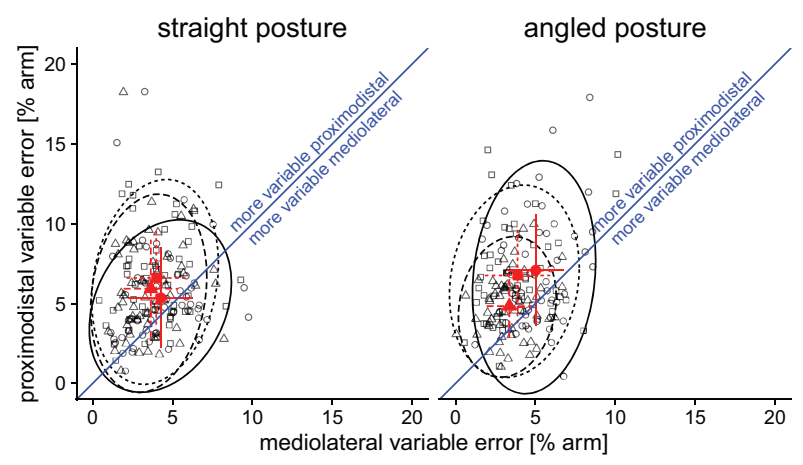

B Final constant error

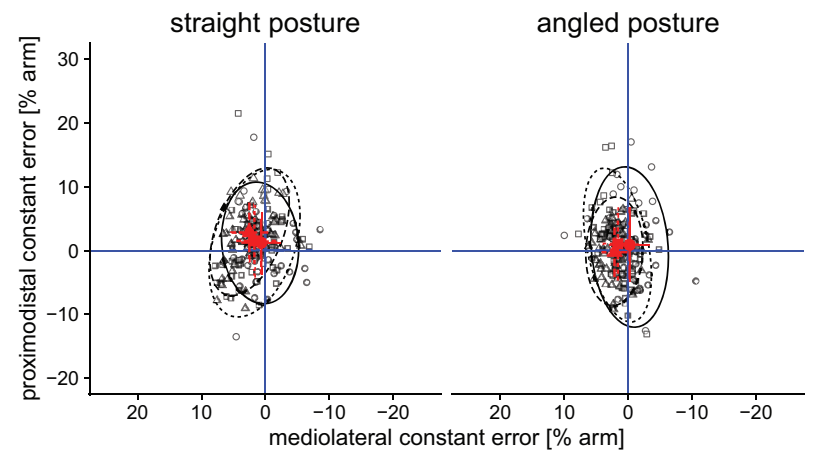

D Final variable error

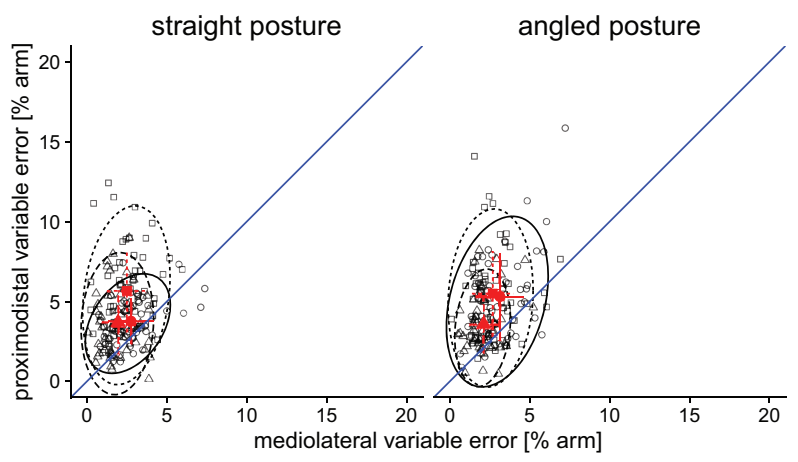

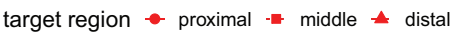

Figure 2. Constant and variable error in Experiment 1. A, B: Constant error in mediolateral and proximodistal direction for the straight and the angled posture for initial localizations. Error was computed as the intrasubject mean localization error over trials, coded as distance from the target, represented by the crossing point of the blue lines. Single participants' error is shown as black points separately for the three target regions (proximal, medial and distal target). The red symbols with crosses indicate group means for the target regions with standard deviations as error bars. The ellipses represent $95 \%$ confidence. The lower-right quadrant of panel a and $b$ indicates bias in distal and lateral direction. (A) At finger touchdown, before search, (B) after search. C, D: Variable error was computed as the intrasubject standard deviation of localization error over trials. Values that fall into the area above the blue diagonal line indicate larger variable errors in proximodistal compared to mediolateral direction. (C) At finger touchdown, before search, (D) after search. See the online article for the color version of this figure.

error. To this end, we first split search trajectories into 20 segments of equal spatial length to control for difference in movement time and speed (see Methods section).

We analyzed error reduction across the 20 segments separately for trials with initially large, initially medium, and initially small localization error at touchdown. Localization error diminished nonlinearly across segments: reduction was greatest during the first search segments, and absent in late segments (Figure 4A and 4B). However, this effect critically depended on initial localization error: The linear term of a polynomial regression was strongly negative for initially large-error trials, indicating continuous improvement in localization over the course of the trial $(b=-7.1$, $p<.001)$. In contrast, it was near zero but slightly positive for initially small-error trials, indicating even slight deterioration of localization over segments $(b=0.90, p<.001)$. Moreover, the quadratic term was strongly positive for the initially large-error trials, indicating that localization improvement was large at the beginning, and absent toward the end of search $(\mathrm{b}=2.4, p<$ .001). In contrast, the quadratic term was small and negative for initially small-error trials, indicating that localization deteriorated at the beginning and remained unchanged at the end of search ( $b=-0.97, p<.001)$. Thus, tactile search significantly reduced localization error at the beginning of search only when participants had set their finger down relatively far from the target. Independent of initial distance to the target, search was ineffective toward the end of search. For trials in which initial localization error was of medium size, both the linear and the quadratic terms fell in between those of the large and small error trials, suggesting a graded effect of initial localization error. The effect that localization error reduced for initial large, but not for small localization error, was present for both arm postures and all three target regions (Figure 4B and Supplemental Table S13 in the online supplementary material). 

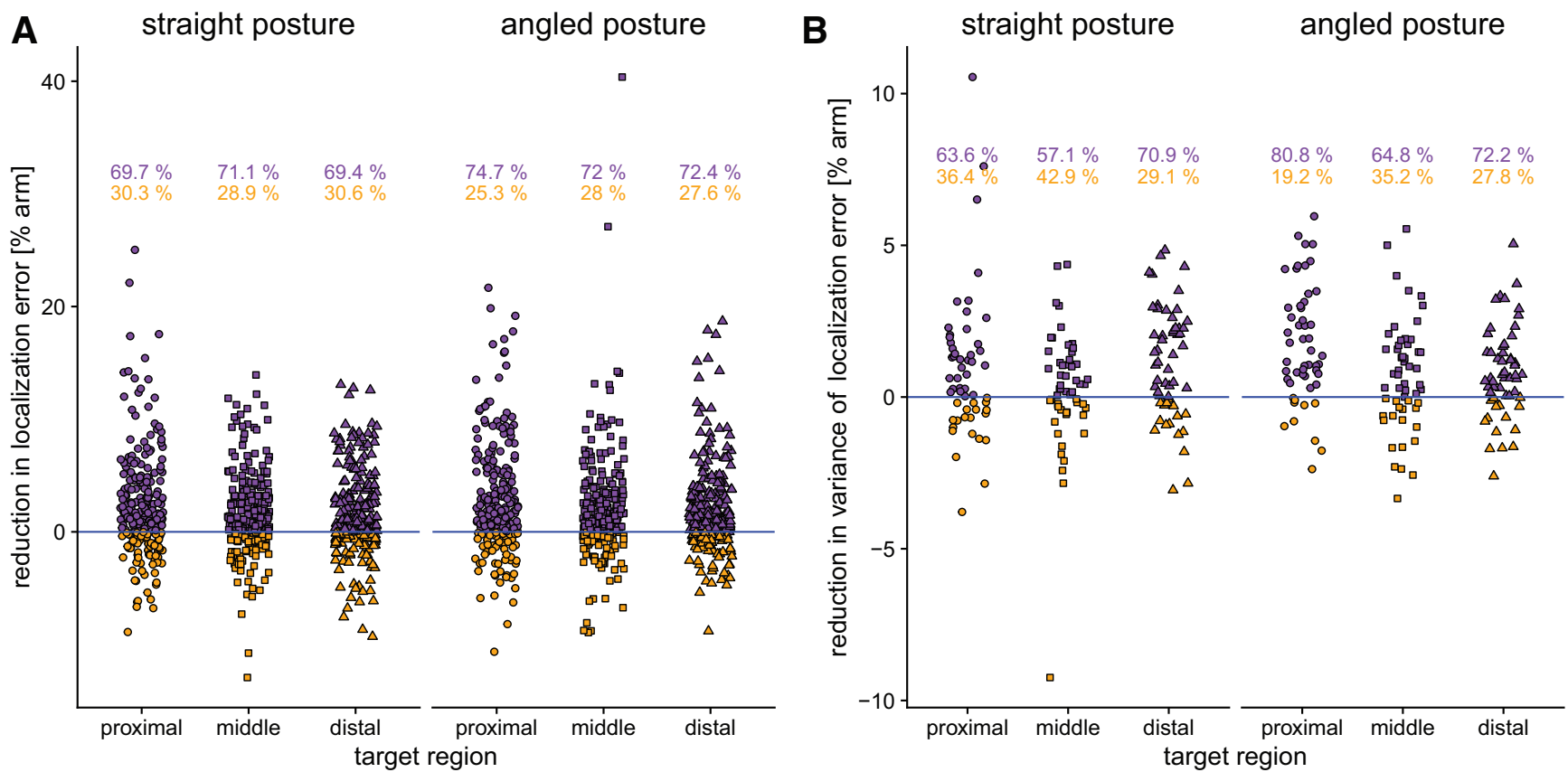

Figure 3. Reduction of direction-independent tactile localization error (computed as Euclidian distance from the target) through search in Experiment 1. A: Intrasubject difference values between localization error before versus after tactile search. Each point represents one trial. B: Difference values between intrasubject variation (computed as standard deviations) of localization error before search versus after search. Positive values indicate smaller variation after search than before, indicating that, through search, localizations gravitate toward their mean value. See the online article for the color version of this figure.

\section{Discussion}

Results of Experiment 1 suggest that search was based on a closed-loop strategy that used online tactile feedback to consistently reduce localization error. However, the absence of localization error reduction in initially small error trials and the later segments of initially large-error trials implies that error reduction is confined to a region that is distant from the target. One possible cause of this limit may be the large size of tactile receptive fields on the arm (Weinstein, 1968). Consistent with this interpretation, we found the average final error (Figure 4D) to be $1-2 \mathrm{~cm}$, which roughly matches existing estimates of tactile localization ability on the arm (Weinstein, 1968).

One alternative interpretation for the reduction in localization error via tactile search, however, is that search is not driven by tactile information provided by the target but is instead achieved by means of motor correction and proprioceptive feedback of the acting, searching arm. Under this notion, participants potentially used a precise tactile target location estimate throughout, but gradually directed their searching finger to that location due to imprecision of the motor system. Experiment 2 tested this idea by comparing localization error when participants were, versus when they were not, allowed to touch the arm during search.

\section{Experiment 2}

\section{Method}

General procedures and experimental design. Participants localized tactile stimuli on the left, extended, ventral forearm with their right index finger either searching on the skin as in Experiment 1 , or on an acrylic glass barrier placed directly above the arm (Longo et al., 2015). This barrier prevented tactile feedback of the target arm while still allowing participants to search for the correct location. Hence, we varied whether participants received tactile feedback from the target surface or not. For both conditions, participants positioned their left arm in a wooden construction $(60 \times$ $39.2 \mathrm{~cm}$, see Figure 5), with the ventral forearm surface turned toward the face. Their elbow rested comfortably on the table surface and the construction maintained the elbow at an angle of $120^{\circ}$. We used two target locations $4 \mathrm{~cm}$ proximal of the center of the wrist that were 1 $\mathrm{cm}$ apart in mediolateral direction. For precise stimulation, we marked the targets on the arm with a pen. Multiple dots were drawn in a $10 \times$ $10 \mathrm{~cm}$ area around the target to obscure for participants the locations that were relevant in the experiment while the experiment was prepared. During the experiment, participants were blindfolded and did not see the drawn locations any longer. The acrylic barrier of $15 \times 15$ $\mathrm{cm}$ was placed above the forearm. It could be attached at a flexible distance, and it was positioned just above the individual's arm without touching it. The barrier was removed for stimulation, and then replaced before localization. Experiment 2 manipulated one single experimental factor: target surface (skin vs. barrier), resulting in two search conditions that were performed in a random order across participants. As in Experiment 1, each target was stimulated and localized five times, resulting in five trials per location, and 20 trials in total per participant $(2$ conditions $\times 2$ target positions $\times 5$ repetitions). As before, participants performed practice trials to become familiar with the procedures. The experiment took about $30 \mathrm{~min}$. 


\section{A}
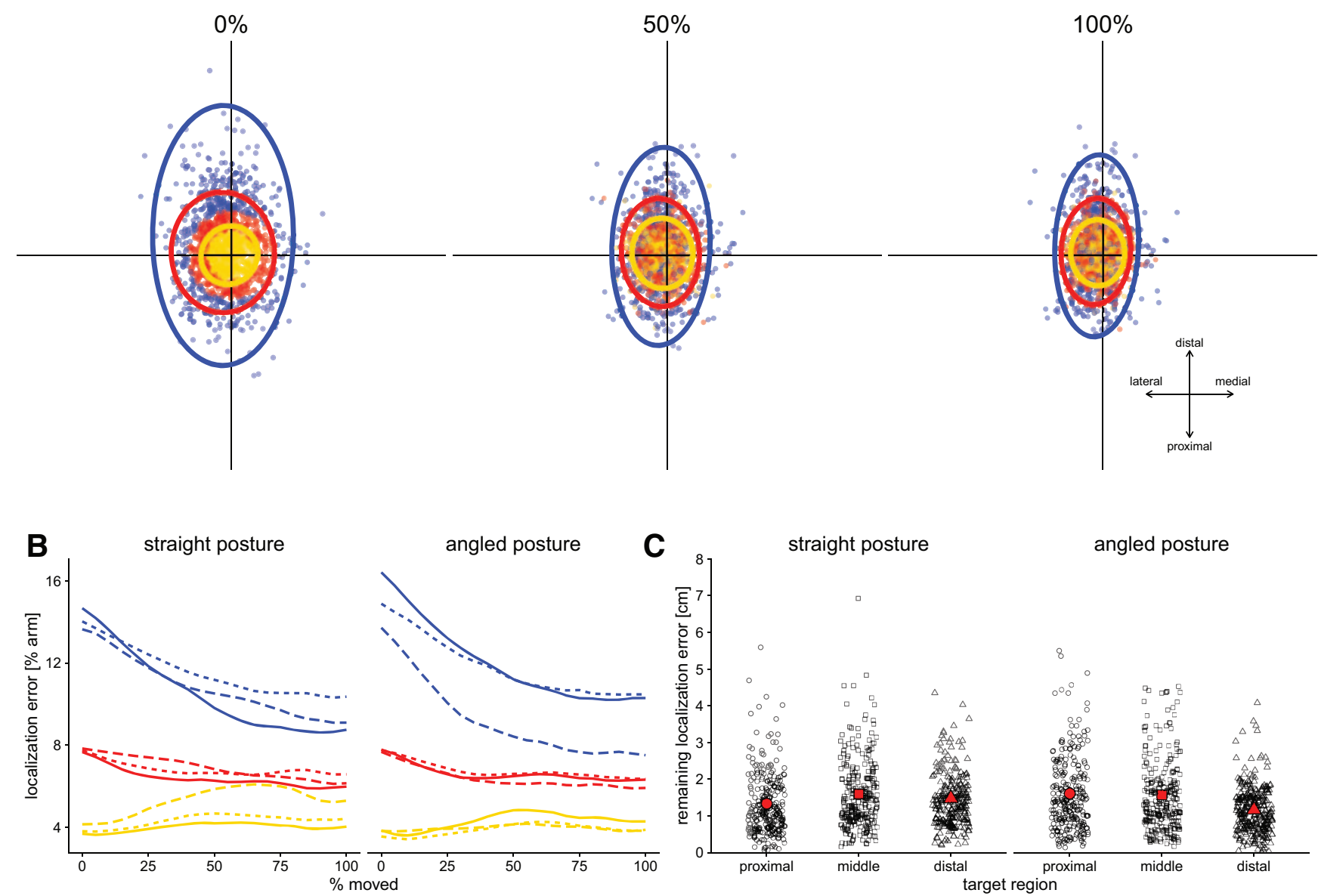

initial localization error $\_$small $\_$medium $\_$large target region - proximal $-\ldots$ middle $\quad$ - dista

Figure 4. A: Distribution of single trial localization error in Experiment 1, pooled across arm postures and target regions. Localization error is defined as the distance of the pointing finger from target position, represented by crossing lines in the middle of the plots. Trials are divided into three subgroups according to initial localization error (blue: large, red: medium, yellow: small) at touchdown. Ellipses represent 95\% confidence for the respective, color-coded trial subgroup. From left to right, at touchdown, after 50\% of the traveled distance, and at end of search. An animated version of this figure is available online as Supplemental Figure S4A in the online supplementary material. B: Change of direction-independent localization error (defined as the Euclidian distance from the target) over the course of tactile search in dependence of initial localization error. C: Remaining direction-independent localization error, in centimeter units, at the end of tactile search. See the online article for the color version of this figure.

The present, as well as the following, control experiments aimed at testing some factors which we had ignored in Experiment 1 in exchange for a naturalistic paradigm. They were therefore performed in the lab. Participants always gave written informed consent, and all experiments were approved by the Ethics Committee of Bielefeld University, Bielefeld, Germany.

Participants. We excluded four of originally 20 tested participants from the analysis due to technical problems during acquisition. The final dataset included 16 participants (aged 19-34, $M=25.4$ years; 8 females). The average arm length was $22.8 \mathrm{~cm}$ (range: $20-25$ $\mathrm{cm})$. Participants took part either without compensation or for course credit. All participants were right-handed, according to the Edinburgh
Handedness Inventory (Oldfield, 1971), and reported to be free of neurological or psychological disorder or any other condition that might affect sensory or cognitive function.

Tactile stimuli. We applied standardized tactile stimulation with a von-Frey filament (Marstock Nervtest, Schriesheim, Germany) with $256 \mathrm{mN}$ force. As in Experiment 1, touch was applied for approximately $1 \mathrm{~s}$.

Tactile localization. The experiment involved a condition in which participants searched on a barrier above their arm, rather than on the arm itself. This barrier had to be removed for stimulation, and then be replaced before search. To keep the time before localization identical for skin and barrier conditions, a timer in- 
A

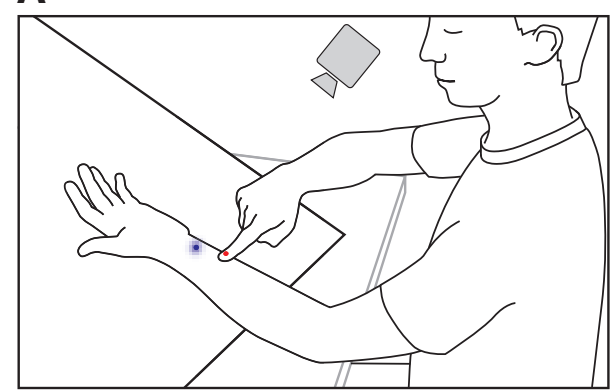

B

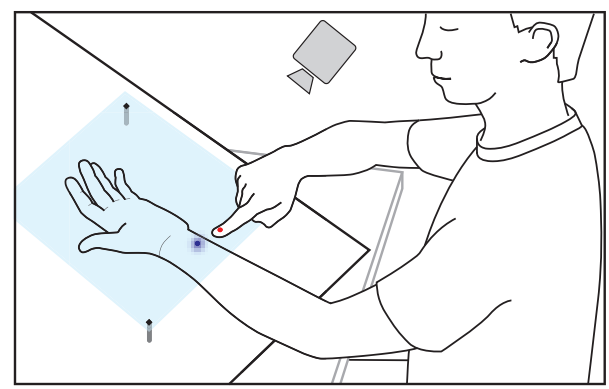

Figure 5. Illustration of procedures used in Experiment 2-4. Touch was applied to the left ventral forearm. The participants localized the stimuli by either moving the finger on the skin (A), as in Experiment 1, or by moving the finger on an acrylic glass barrier (B), preventing tactile feedback from the skin of the target arm. See the online article for the color version of this figure.

structed participants to refrain from initiating the search in both conditions. To keep demands on tactile memory to a minimum, we chose a duration of $3 \mathrm{~s}$, which was the shortest interval that was practically feasible.

Estimation of sample size and power. We estimated the required participant number to replicate the reduction of localization error from before to after search observed in Experiment 1. We drew one to five trials from the data of Experiment 1 for simulated samples of 10-58 participants. We restricted our draws to the target region and arm posture of Experiment 1 that most closely resembled the test conditions of Experiment 2. Drawn samples were not replaced. For each number of trials and sample size, we ran 250 simulations and computed a linear mixed model to test, for each repetition, whether the simulated data set contained a significant difference in localization error before and after tactile search. The percentage of significant results within a given set of 250 simulations represents the power of the simulated experiment. Simulation results indicated that power was higher than $95 \%$ with 20 participants, and above $90 \%$ with 16 participants, when conducting 5 localization trials per condition. We measured 20 participants, providing us with sufficient power even with some data loss (which we expected, given our experience from Experiment 1). From the 20 participants we measured, four participants could not be analyzed, and we did not replace them given that power was sufficient.

Data loss and exclusion. At least one trial was missing in 12 out of the 16 participants $(75 \%)$, resulting in overall data loss of $9 \%$. The presented analysis is based on 291 trials.

Data acquisition and extraction. The methods for data capture and extraction of trajectories were identical to those of Experiment 1. The barrier in Experiment 2 was transparent and allowed recording the arm and the locations marked on it during search.

Data preprocessing. Preprocessing was performed as in Experiment 1 , with the only difference that the anatomical landmarks differed. The camera's field of view was smaller than in Experiment 1 and excluded the elbow. The four anatomical landmarks defined during analysis were therefore the inside and outside of the wrist (as in Experiment 1) and inner and outer border of the forearm at the midpoint between the wrist and the elbow, which had been marked on the arm using ink, and could therefore be identified in the images.
Statistical analysis. Analysis methods were analogous to those of Experiment 1. However, the analysis included the fixed factors surface (skin, barrier), direction (proximodistal and mediolateral), and search (initial and final localization).

\section{Results}

Constant localization error. We observed a three-way interaction expressing that constant error depended on target surface, direction and search, $F(1,157.7)=17.9, p<.001$.

On the skin, there was no significant bias in either direction before search; after search, bias increased significantly $(p=.002)$, resulting in a significant bias in the medial direction $(-2.9 \%$ arm length, $p=.007$; Figure 6B and Supplemental Table S15 in the online supplementary material).

On the barrier, there was a strong bias in the lateral direction, which was of comparable size before and after search $(11.5 \%$ vs. $10.5 \%$ arm length, both $p<.001)$. However, there was a slight increase of proximal bias through search from before (4.5\% arm length, $p=.037)$ to after search (9.5\% arm length, $p=.009)$.

In the analysis of Euclidian distance in units of centimeters, the average localization error for barrier search was $4.5 \mathrm{~cm}$ before, and $4.4 \mathrm{~cm}$ after search, and, thus, nearly three times larger than the remaining error of $1.5 \mathrm{~cm}$ for skin search (Figure 6D).

Variable localization error. Variable error generally decreased with search, $F(1,233)=22.1, p<.001$. However, it was larger on the barrier than on the skin, $F(1,233)=131.9, p<.001$, Figure $6 \mathrm{C}$, and it was larger in the proximodistal than in the mediolateral direction, $F(1,233)=55.2, p<.001$. This latter difference was more pronounced on the barrier than on the skin, $F(1,233)=14.5, p<.001$.

Progression of error reduction in trajectories. Search on the skin reduced localization error, especially in the first half of search (linear term: $\mathrm{b}=-8.7, p<.001$; quadratic term: $\mathrm{b}=3.8$, $p<.001$; Figure 6A and 6C). Error reduction depended on initial localization error and was strongest in trials with initially large localization error, whereas it was weak and not significant when initial localization error was small, $F(2,6028)=188.0, p<.001$ (see Supplemental Tables S18 and S19 in the online supplementary material). For barrier search in the 20-segment model, the linear term was significantly smaller and with reversed sign, indi- 

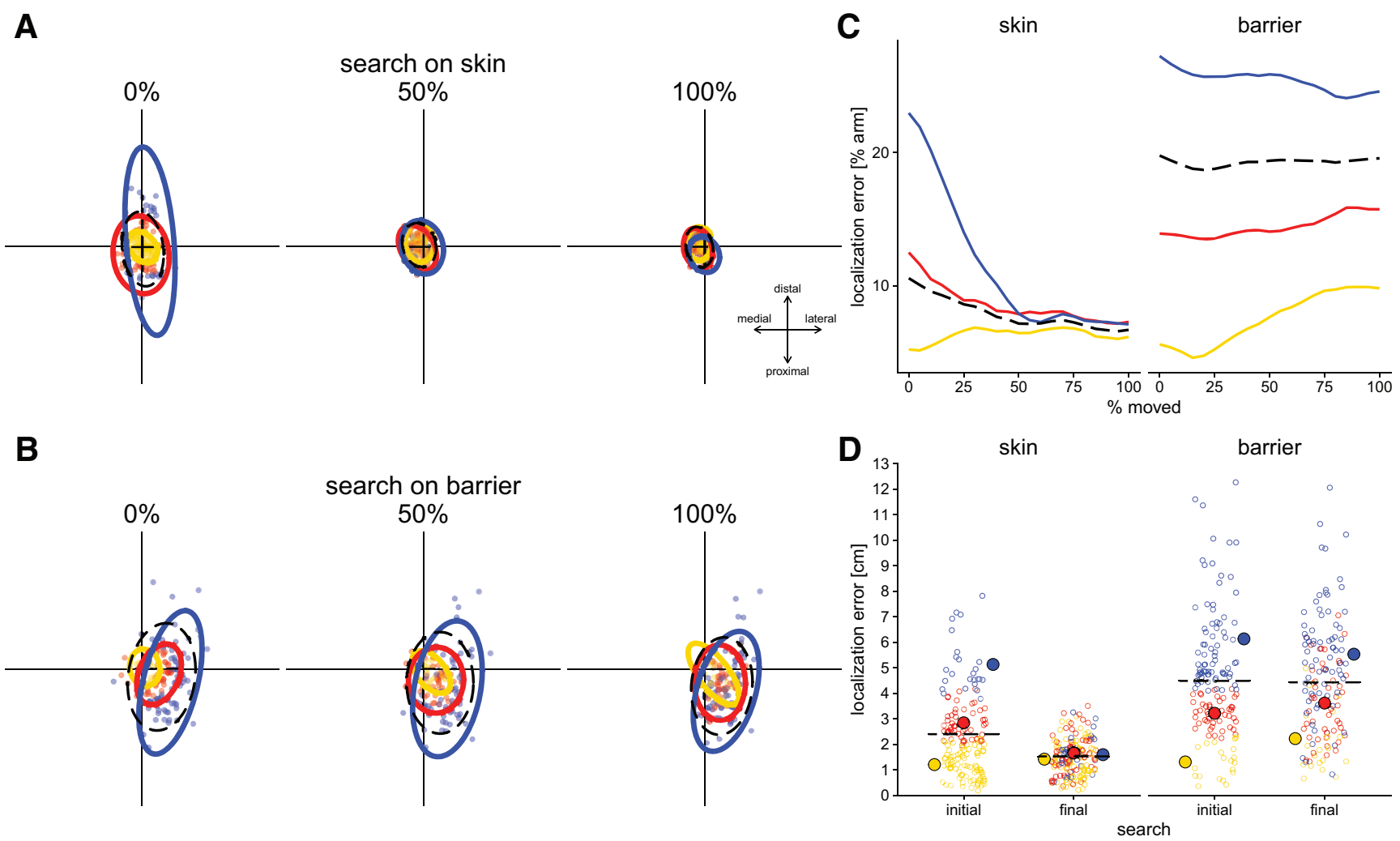

initial localization error $\square$ small $\square$ medium $\square$ large

Figure 6. Results of Experiment 2. A: Localization error distribution of single trials for search with tactile feedback, comparable to Experiment 1 (compare to Figure 4A). B: Localization error distributions for search on an acrylic barrier, that is, without tactile feedback on the target arm. An animated version of (A) and (B) is available online as Supplemental Figure S6A and 6B in the online supplementary material. C: Change of localization error over the course of search depending on initial distance from the target for tactile feedback and acrylic barrier conditions. D: Remaining distance from the target after search in centimeter units. Colors code subgroups of trials with different initial distance from the target; the dotted lines indicate the average of all trial types collapsed. See the online article for the color version of this figure.

cating a slight increase of localization error $(b=2.2, p<.001)$; the quadratic term was not significant $(b=0.4, p=.445)$. Notably, also on the barrier, the linear slopes varied depending on initial error indicating increasing localization error when the initial error was small, and decreasing localization error when initial error was large (Figure 4B and 4C and Supplemental Table S19 in the online supplementary material).

In relative terms, these results reflect an improvement of merely $1 \%$ for search on the barrier and, on average, $36 \%$ for search on the skin (initially large/medium/small-error: $-17 \% / 41 \% / 69 \%$ ).

\section{Discussion}

Experiment 2 replicated Experiment 1 in that search on the skin improved tactile localization and that this improvement only occurred with initially large localization error. Importantly, error reduction by search was absent when participants could not produce tactile input on their target arm because they searched on a barrier. Experiment 2, therefore, supports the notion that tactile feedback from the target area is necessary for localization im- provement during active search. We conclude that closed-loop tactile information on the target region, but not proprioceptive information of the acting arm, considerably improved tactile localization accuracy and precision.

Experiment 2 also complements Experiment 1 with respect to observed localization biases. In contrast to Experiment 1 and in line with previous studies (Mancini, Longo, Iannetti, et al., 2011), we did not observe any distal constant error (all $p>.05$; see Supplemental Table S15 in the online supplementary material) on the ventral as opposed to on the dorsal forearm.

There were some experimental parameters in which Experiment 2 differed from Experiment 1 besides the prevention of tactile feedback. First, we chose the ventral instead of the dorsal forearm as the stimulation site. The replication shows that the observed effect generalizes to the ventral forearm. Second, localization in Experiment 2 was delayed and might therefore be more "offline" as compared to Experiment 1 (Gallace \& Spence, 2009). The replication of improvement by search, therefore, demonstrated that search is not confined to immediate, "online" localization. 
Yet, a potential alternative explanation for the improvement by target-related tactile input remains: So far, we always encouraged participants to search on the skin. These instructions may have reduced participants' motivation to aim precisely for their initial reach that set down the searching finger on the target arm. Accordingly, our instructions may have led to large initial error that does not reflect the upper limit of participants' ability to indicate a tactile location with pointing only. Instead, the initial error observed in the two previous experiments may reflect a strategy that trades off the effort of aiming during flight against the expected (presumably lower) effort to correct the resulting error during tactile search. Under this notion it is possible that participants accurately represented the actual target location and that improvement by search did not reflect a true localization benefit gained from online tactile feedback, but rather a post hoc correction of a strategic motor error. We addressed this potential confound in Experiment 3 by instructing participants either to localize by search, as before, or to perform the best possible reach toward the target location without any option to search on the skin thereafter.

\section{Experiment 3}

\section{Method}

General procedures and experimental design. The general procedures were identical to those of Experiment 2. The experimental factors were target surface (skin vs. barrier) and instruction (search vs. no search), resulting in four conditions that were performed blockwise in a random order across participants. With the "search" instruction, participants were allowed to search following touchdown, as in Experiments 1 and 2. With the "no search" instruction, participants had to aim their reach as precisely as possible and to touch down on the target arm, but they were not allowed to correct thereafter. Similar to Experiment 1, we used three target regions to prevent adaptation effects. The regions were positioned along the medial line of the arm: about $4 \mathrm{~cm}$ proximal of the wrist, about $4 \mathrm{~cm}$ distal from the position half-way between the elbow crook and the wrist, and halfway between the two. As previously, stimulus location varied slightly from trial to trial; however, and target position was recovered from video. Within each condition, stimulation occurred at the three possible locations in pseudorandom order. Each target location was tested three times. Experiment 3 therefore comprised 36 trials per participant (4 conditions $\times 3$ target positions $\times 3$ repetitions) and took about $60 \mathrm{~min}$.

Participants. We recruited 20 participants. Data of one participant was excluded due to technical problems during acquisition. The resulting sample consisted of 19 participants (aged 19$62, M=28.3$ years; 14 females). The average arm length was 25.4 cm (range: 22-29 cm). All participants were right-handed. All procedures were identical to Experiment 2 concerning selection, exclusion, credit, and written, informed consent.

Tactile stimuli. Stimulation was applied with a pressurestandardized $256 \mathrm{mN}$ von-Frey filament.

Tactile localization. Participants were blindfolded during stimulation and localization. Localization proceeded as in Experiment 2. In the "no search" condition, participants rested with their finger at the same position after touchdown for some time before lifting off again.

Estimation of sample size and power. We determined sample size with simulations as in Experiment 2, based on 9 trials per condition that were pooled across the three target regions. We report details of the simulations in the online supplementary material.

Data acquisition, extraction and preprocessing. We recorded 30 frames/s with a conventional webcam (Logitech C270, Logitech, Newark, CA) controlled via a Windows 7 PC using PsychoPy (v3.2.4), with a resolution of $800 \times 600$ px. All other methods as well as image and data preprocessing were the same as before.

Data loss and exclusion. We could not use 90 trials (13\%) across the entire sample. The presented analysis is based on 594 trials.

Hypotheses and statistical analysis. We based analysis on Euclidian distance from the target. Our analysis focused on three aspects:

First, if participants in our previous experiments had used lowerthan-possible precision for their initial reach to the target arm, then participants in Experiment 3 should exhibit smaller initial localization error - that is, localization error at touchdown-when they are not allowed to actively search after the reach. Accordingly, initial error should be larger when search is allowed.

Second, however, even if the difference in instruction were to reduce the initial error, participants may still be able to improve localization through active search (when allowed) above and beyond their highest-effort initial localization. Thus, final localization after active search may be better than initial localization without active search, and such a result would indicate that tactile feedback in an closed-loop situation improves tactile localization beyond what can be achieved through reaching alone.

Finally, given the results of Experiment 2, we expected that search - if at all-should be effective only on the skin, but not on the barrier.

The factorial design of Experiment 3 is incomplete: the "no search" condition comprises only an initial localization error, but not a final localization error, because improvement by active search was not allowed by instruction. For LMM analysis, we, therefore, created a fixed factor localization that contained all localization conditions across the two instructions (no search: error at touchdown; search: initial error at touchdown, final error after search). To test for specificity of search on the skin, we included the fixed factor surface (skin, barrier).

\section{Results}

There was a significant main effect of localization, $F(2,30.2)=$ 26.6, $p<.001$; we compared pairs of conditions using estimated marginal means analysis. When participants were allowed to search on the skin, localization error was smaller after $(11 \%$ arm length) than before search (17\% before, difference: $p<.001)$; this result replicated the previous findings from Experiments 1 and 2 . Importantly, initial localization error was indistinguishable between the two instructions; in other words, initial error was similar whether participants were allowed to correct afterward or not $(17 \%$ vs. $16 \%$ arm length, $p=.436$ ). Moreover, final error when participants were allowed to search was smaller than the reaching 
error when they were not allowed to correct (11\% vs. $16 \%$ arm length, $p<.001)$.

Localization error was significantly larger on the barrier than on the skin, $F(1,17.8)=61.65, p<.001$. As for the skin, localization error of the initial reach did not differ between the two instructions (40\% vs. $39 \%$ arm length, $p=.627$ ). Yet, participants improved localization error by search also when localizing on the barrier, indicated in a lack of interaction between localization and surface, $F(2,848.5)=0.125, p=.883$ (see Figure 7 and Supplemental Tables S20 and S21 in the online supplementary material) and a significant comparison of error before and after barrier search (40\% arm length before vs. $34 \%$ after search, difference: $p<$ .001). It is noteworthy, however, that the initial localization error on the barrier was much larger than in Experiment 2. Therefore, even though participants improved by barrier search, their final error was still considerably larger than that observed in Experiment 2 .

\section{Discussion}

Experiment 3 showed that the improvement in localization through search on the target surface is not an artifact of task instructions. Participants' initial localization performance was comparable when they were or were not allowed to search after touchdown. More importantly, search reduced localization error compared to initial localization of both the "search" and the "no search" instructions. These results rule out that the improvement observed in Experiments 1-3 resulted from strategically reduced effort or precision of the reach that brings the searching finger near the tactile location on the target arm, based on the consideration that such reduced precision could be corrected in the following search. In fact, the present result suggests that the initial localization in the "search" instruction of all three experiments is an accurate estimate of localization performance without online tactile feedback.

It is noteworthy that localization on the barrier was overall worse in Experiment 3 than in Experiment 2 ( $\sim 40 \%$ vs. $\sim 20 \%$ arm length). Setup, camera position, and analysis were identical in the two experiments and cannot, therefore, explain the performance difference. One possible explanation is that the different instructions of Experiment 3 led participants to point more spontaneously and, as a consequence, less accurately. In fact, there was a strong bias in the direction from which the pointing movements originated (see in Figure 7B, lower panels; note the much larger localization errors in the upper right corner of the distributions). This error pattern is compatible with the notion that participants were actually aiming at their arm, and that their movement was stopped unexpectedly by the barrier in flight. In line with this interpretation, 12 participants commented on the barrier condition as being particularly difficult due to the spatial gap between the arm and the barrier impeding localization and interrupting pointing movements. Because of the high initial error, participants presumably noticed that their location was inaccurate and then compensated by corrective movements, explaining the unexpected improvement in the
A

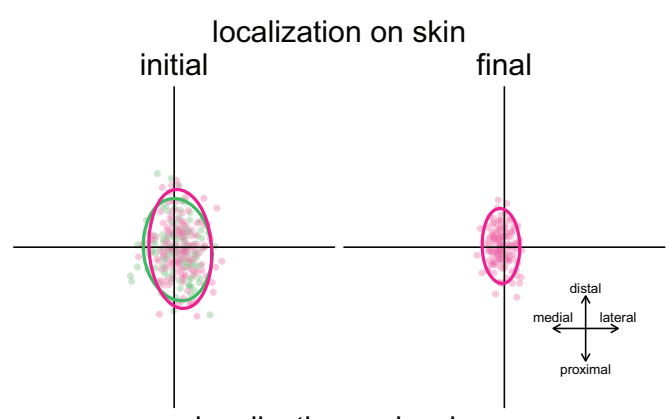

B
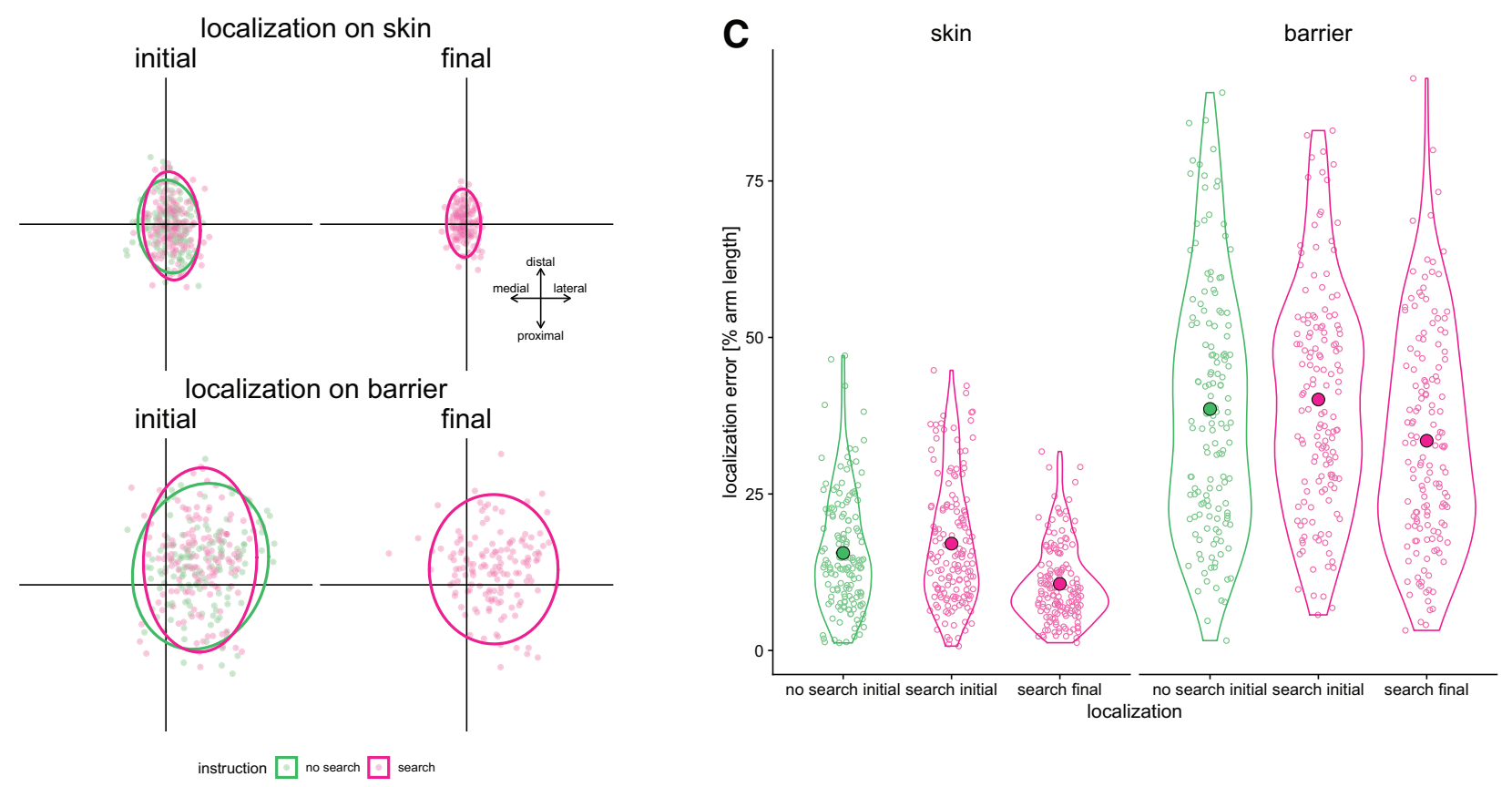

Figure 7. Results of Experiment 3. Localization error distribution of single trials for initial localizations in the "no search" and "search" instruction and final localization in the "search" instruction on the skin (A) and on the acrylic barrier (B). An animated version of Panels A and B is available online as Supplemental Figure S7A and 7B in the online supplementary material. C: Single trial distributions for the same conditions as in a and b, expressed as Euclidian distances from the target position. The large, solid points represent mean values. See the online article for the color version of this figure. 
barrier condition. Recall, that in Experiment 2, too, participants had benefitted from online correction on the barrier when their initial localization had been in the worst third of trials. In Experiment 3, in many trials the initial localization error was as large as the largest errors in Experiment 2, likely explaining the unexpected improvement in Experiment 3's barrier condition. Notably, because Experiment 2 had shown that barrier search did not improve localization when initial error was small, the large final error observed in Experiment 3's barrier condition suggests that it was not the tactile feedback created on the barrier that improved localization, but that, instead, this improvement was based on proprioceptive feedback.

In the three experiments we have presented, participants had no visual information during touch and localization. When participants cannot search, therefore, they must rely on proprioception to direct the reaching arm to the target arm. There are, therefore, two possible mechanisms that may account for the localization improvement by search we have reported. First, improvement may be based on comparison of the tactile target with the self-induced tactile information; according to this idea, improvement would be unique to tactile search. Second, however, localization improvement may be based on improved information of the relative location of the reaching arm and the target arm. It is well known that proprioception is imprecise (Fuentes \& Bastian, 2010; Risi, Shah, Mrotek, Casadio, \& Scheidt, 2019), and vision strongly influences our estimate of where the hand is in space (Risi et al., 2019; Smeets, van den Dobbelsteen, de Grave, van Beers, \& Brenner, 2006). Under this notion, online tactile feedback during active search may provide information that helps estimating the spatial relationship between effector and target. This update could then be used to guide the goal-directed movement toward the target. Accordingly, improvement would not be unique to tactile search. We note that the total improvement by tactile search may also be a compound of the two accounts, that is, vision may improve tactile localization compared to a blindfolded no-search context, but tactile search may further improve localization performance.

Experiment 4 tested these different possibilities by either preventing or allowing visual control during active tactile search.

\section{Experiment 4}

\section{Method}

General procedures and experimental design. Participants closed their eyes while the tactile stimulus was applied. They were then allowed to search for the target while their eyes were either open or closed. With eyes open, participants received visual information about the spatial relationship between the limbs while reaching toward the target arm and during tactile search. We expected that visual control may improve tactile localization, but that tactile feedback may allow further, touch-specific localization improvement. To increase sensitivity for a role of both factors, participants performed tactile localization both on the arm and on the barrier; here, visual but not online tactile feedback is available for tactile localization. Experiment 4, thus, had two experimental factors: target surface (skin vs. barrier) and vision (eyes closed vs. eyes open), resulting in four conditions that were performed blockwise in a random order across participants. We used the same three target regions along the medial line of the ventral forearm as in
Experiment 3. Within each condition, stimulation occurred at the three possible locations in pseudorandom order. Each target location was tested five times. Experiment 3, therefore, comprised 60 trials per participant $(4$ conditions $\times 3$ target positions $\times 5$ repetitions) and took about $90 \mathrm{~min}$.

Participants. We recruited 20 participants (aged $18-33, M=$ 22.5 years; 18 females). All participants were right-handed. The average arm length was $25.4 \mathrm{~cm}$ (range: 22-29 cm). All procedures were identical to Experiment 2 and 3 concerning selection, exclusion, credit, and written, informed consent. No participant was excluded from analysis.

Tactile stimuli. During piloting, we observed that the $256 \mathrm{mN}$ von-Frey filament used in Experiment 2 and 3 can leave a tiny but visible mark on the skin that participants could see when their eyes were open. We therefore applied touch following the procedure of Experiment 1, using the blunt wooden stick that left no visual marks on the skin.

Tactile localization. For the visual conditions, participants opened their eyes before starting their reach toward the target arm. For the eyes-closed conditions, they kept their eyes closed throughout the entire block.

Estimation of sample size and power. We estimated power using simulations as before (see the online supplementary material).

Data acquisition, extraction and preprocessing. Data was acquired and processed as in Experiment 3.

Data loss and exclusion. Procedures were identical to Experiment 3. In total, we excluded 39 trials (3\%). The analysis is based on 1161 trials.

Hypotheses and statistical analysis. We confined analysis to Euclidian distance from the target. The experimental factors were surface (skin, barrier), vision (eyes open, eyes closed), and search (initial and final localization). We focused on three aspects. First, we expected to replicate the effect of search on the skin with the eyes closed. Second, if tactile search had any benefit over spatial information provided by vision, then localization error on the skin at the end of active search should be smaller than for initial localization with open eyes. Note, participants can correct based on tactile feedback also in the "eyes open" condition; therefore, final localization error should be indistinguishable for search with and without vision. Finally, vision should provide reliable feedback also in the barrier condition and likely lead to reduced initial and final localization error when localizing on the barrier. Blindfolded search on the barrier should be comparable to the results of Experiments 2 and 3, that is, participants should either exhibit large initial error and large final error despite some improvement (as in Experiment 3), or they should exhibit no improvement at all through search (as in Experiment 2).

\section{Results}

Participants benefitted from tactile search both on the skin and on the barrier, $F(1,21.7)=30.4, p<.001$, but only when vision was prevented, $F(1,22.8)=16.8, p<.001$ (Figure 8A-C). Furthermore, vision had a larger effect in the barrier than in the skin condition, $F(1,19.0)=52.7, p<.001$. To follow up these interactions, we further analyzed the estimated marginal means for skin and barrier search separately, based on the above-stated hypothesis that behavior should differ between the two conditions. 

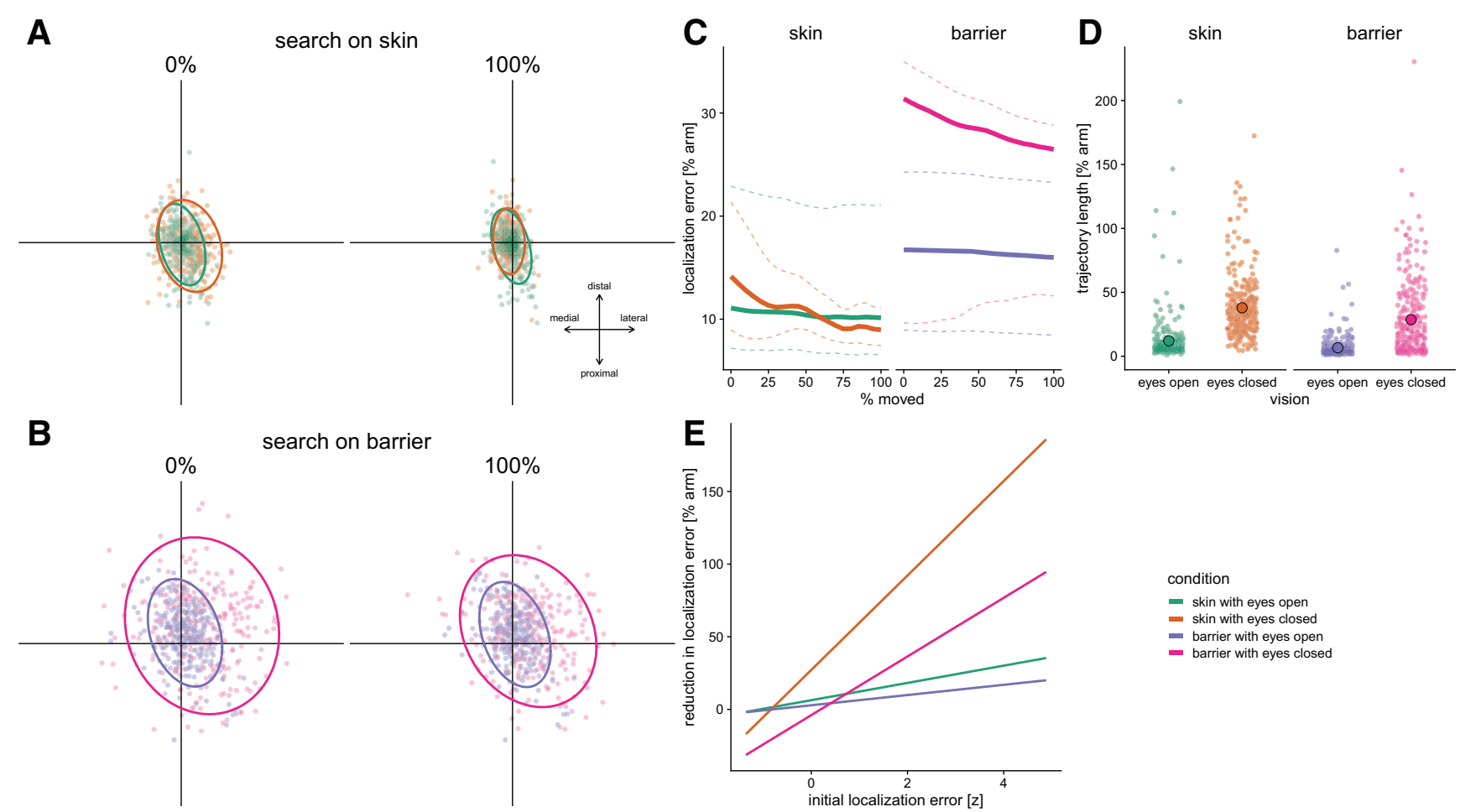

condition

- skin with eyes open - skin with eyes closed - barrier with eyes closed

Figure 8. Results of Experiment 4. Localization error distribution of single trials for initial and final localizations in the "eyes open" and "eyes closed" conditions on the skin (A) and on the acrylic barrier (B). C: Change of localization error over the course of search depending for the conditions. The thick solid lines represent the mean values for the condition; the thin dashed lines correspond to trials with larger (above median) and small (below median) initial localization error. D: Distribution of single trials' search trajectory length for the conditions. E: Regression lines expressing the relationship between initial localization error and reduction of localization error through search. Regression coefficients result from a linear mixed model taking account the repeated-measures structure of the data (see test for details). See the online article for the color version of this figure.

Search on the skin. When vision was prevented, localization error on the skin reduced from $14 \%$ to $9 \%$ arm length $(p<.001)$. No such improvement was evident when participants searched with open eyes (from $11 \%$ to $10 \%$ arm length, $p=.236$ ). However, the initial localization error was significantly smaller with open than with closed eyes ( $11 \%$ vs. $14 \%$ arm length, $p=.033$ ), suggesting that visual information partly compensated for the improvement observed previously for tactile search. Yet, localization without vision resulted in smaller final localization error than initial localization with vision (9\% vs. $11 \%$ arm length, $p=.043$; Figure $8 \mathrm{C}$ ), even if the absolute size of this additional improvement unique to tactile search was small $(0.52 \mathrm{~cm})$.

As expected, final localization error was comparable both after searching with and without vision (10\% vs. $9 \%$ arm length, $p=$ .236).

Search on the barrier. As for the skin, localization error significantly decreased from before to after search (32\% vs. $27 \%$ arm length, $p<.001$ ) with closed eyes, but not with open eyes ( $17 \%$ vs. $16 \%$ arm length, $p=.340)$. In contrast to search on the skin, vision had a strong general effect in the barrier condition. Localization error was significantly smaller with than without vision both before $(17 \%$ vs. $32 \%$ arm length, $p<.001)$ and after search ( $16 \%$ vs. $27 \%$ arm length, $p<.001$; see Figure 8 C). Hence, in contrast to the skin, there remained a large difference between vision and no vision also for the final localizations. Thus, search on the barrier did not improve localization near as much as visual feedback and, accordingly, there was no additional benefit of tactile search on the barrier as was evident on the skin.

\section{Exploratory Analyses}

The lack of improvement by search with vision, both on the skin and on the barrier, was an unexpected result. We performed several analyses to explore potential explanations of the observed result pattern. There are two possible explanations for this finding: First, participants may simply not have made use of the option to tactually search after having touched down guided by vision. Second, they may have searched, but their search was ineffective. To distinguish between these possibilities, we compared trajectory lengths between visual and nonvisual tactile search (see Figure 8D). Trajectories were longer, suggesting increased search activity, when searching on the skin, $F(1,18.9)=7.7, p=.012$. Importantly, irrespective of the surface, trajectories were significantly shorter with open than with closed eyes, $F(1,19.0)=49.6$, $p<.001$; see Supplemental Table S25 for trajectory lengths. This suggests overall less search activity with open eyes, in line with the 
first explanation. In fact, in many trials, participants made almost no corrective movements at all, despite the explicit encouragement to search during task instructions (Figure 8D), and in stark contrast to their behavior when vision was not available.

One possible reason for this result is that participants may search less under visual control because the initial localization error is very small. Under this notion, humans perform visually guided reaching to a touched location on the skin quite proficiently, usually making tactually guided correction unnecessary. However, one should expect that in the case of larger reaching error, discrepancy between target and searching finger would be detected based on the mismatch between the remembered and the self-induced tactile input generated by touching down with the reaching finger. Accordingly, for Experiment 4 we expected that search should be more prevalent, and lead to larger improvement, when the initial localization error at touchdown under visual guidance was large. We ran a linear mixed model that tested whether the initial localization error predicts the improvement by search, defined as the difference between initial and final localization error (Figure 8E). Indeed, consistent with the results of Experiments 1 and 2, improvement was higher in trials with larger initial errors when participants searched with their eyes closed. For search on the skin this relationship was significantly stronger than in all other conditions, including barrier with closed eyes (slope between initial error and improvement by search: 9.7, $p<.001$ vs. $6.0, p<.001$, difference: $p<.001$ ). With vision, the slopes were much smaller $(1.8, p=.002$ for skin and $1.1, p=.025$ for barrier) and did not differ significantly from each other $(p=.314)$. In other words, even when initial error was large, participants did not correct this error as efficiently when vision was compared to when it was not available. This pattern was surprisingly consistent across participants and not just driven by a small subset of participants (see within-participant correlations in the online supplementary material)

\section{Discussion}

Experiment 4 produced four important results. First, when vision was unavailable, participants were able to improve tactile localization by active tactile search. Thus, we replicated our initial result a third time. Second, when vision was available, tactile localization was markedly improved already at touchdown, suggesting that visual information guides the reaching arm with high spatial precision, almost as effectively as tactile search on the skin. Third, tactile search further improved tactile localization, albeit only when participants did not have vision available. Fourth, participants refrained from searching when they had visual information, resulting in (slightly) worse final localization when localizing on the skin with rather than without vision.

We will address the first three results in the General Discussion and briefly turn here to the unexpected, suboptimal localization performance under visual guidance. Maybe the most surprising result with respect to visually guided tactile localization is that participants did not consistently correct their initial localization even when their initial error was large. The observation that final localization was best when participants localized without vision, and that there was a strong relationship between initial error and improvement during search without vision shows that actively created tactile input would have been available to improve perfor- mance. The finding that this information was not used is surprising and appears to contradict the principle that simultaneously available sensory spatial estimates are combined in a statistically (near-) optimal way across modalities (Alais \& Burr, 2004; Ernst $\&$ Banks, 2002). There are at least two potential explanations for the present finding. First, participants may have very high confidence in their visual judgments, preventing further corrections even if the tactile system signals remaining error. Within the framework of optimal integration, vision may receive a considerably stronger weight than touch because of its high spatial reliability in other, nontactile tasks that make up the majority of our interaction with the world. After all, the remaining tactile localization error, even without vision, is in the order of $1-2 \mathrm{~cm}$, a spatial resolution that is orders of magnitude worse than that of vision. Second, visual information may be attentionally prioritized, leading to down-weighting of tactile input. This explanation seems at odds with findings of visual enhancement of touch that suggest boosting of tactile input when one looks at the touched body part (Haggard, Taylor-Clarke, \& Kennett, 2003; Press, Taylor-Clarke, Kennett, \& Haggard, 2004; Spence, Nicholls, Gillespie, \& Driver, 1998; Taylor-Clark, Kennett, \& Haggard, 2002). However, it is consistent with the naturally occurring behavior of closing one's eyes when trying to pay close attention to a tactile sensation.

\section{General Discussion}

Human perception of touch location is subject to multiple biases and illusions (Longo et al., 2015; Mancini, Longo, Kammers, \& Haggard, 2011). These phenomena are related both to basic sensory processes such as the shape of receptive fields and low tactile receptor density of many body regions (Longo \& Haggard, 2011; Weinstein, 1968) and to a multitude of cognitive factors (Badde \& Heed, 2016; Taylor-Clark et al., 2002). Notably, virtually all methods that are currently employed in tactile localization and body representation research deliberately prevent tactile feedback of the target region, presumably to focus on "pure" perceptual processing. This approach has significantly advanced our understanding of human body representations. Yet, open-loop procedures neglect he active nature of perception; therefore, their use has potential downsides. Open-loop procedures likely overestimate localization error; they may identify representational formats that lack a relation to behavior; and, by design, they are prone to overlooking interactions of sensory and perceptual with motor functions. Accordingly, experimental results based on isolating perceptual or representational aspects of tactile-spatial processing should be carefully compared with results from paradigms that engage natural perception-action loops.

Here, we presented the first comprehensive series of experiments that attempts a dedicated comparison of open-loop and closed-loop tactile localization. Beyond the many detailed aspects discussed in the respective Discussion sections of each experiment, this study revealed four important general results: First, active tactile search, which produces tactile feedback from the tactile target area, significantly improves tactile localization. This result suggests that closed-loop tactile-motor behavior leads to more accurate localization than open-loop behavior. Second, only a fraction of this improvement is unique to tactile search, and similar localization improvement can be achieved through visual information. Third, localization on a 
barrier directly above the arm-that is, very close to the tactile target area-obstructed tactile localization performance, resulting in largely inflated estimates of constant and variable localization error as well as altered corrective behavior. Fourth, even if our experiments only addressed one particular localization behavior, the systematic biases and distortions we observed under closed-loop localization matched those reported with other, open-loop localization methods. We will discuss each of these four points in the following sections.

\section{Active Tactile Search on the Target Limb Improves Tactile Localization}

Actively moving one's finger along the surface of the skin produces tactile feedback on the target region that can potentially be used to refine localization behavior by means of a comparison of the online tactile information with that produced by the remembered target stimulus. In all four experiments, participants indeed improved localization when they searched on the target arm's skin. This beneficial effect of tactile search generalizes over different target areas, namely multiple regions on the dorsal and ventral forearm. The effect also generalized over posture and, accordingly, the movement direction of the searching arm, evident in comparable benefit with straight and angled target arms. Finally, the search benefit persisted when the tactile stimulation had to be remembered over several seconds before the reach to the arm, and with it also the tactile search, could begin. This latter finding implies that the search benefit may be independent of different forms of spatial memory, given that "online" and memory-based localization may rely on different cognitive processes and brain regions, respectively (Gallace \& Spence, 2009; Króliczak, Heard, Goodale, \& Gregory, 2006). It is, furthermore, of note that participants appear to have pointed with the highest possible precision even when instructions allowed them to trade off accuracy of the initial reach with search on the skin.

However, although participants used their highest possible precision for their initial reach, their tactile search was nonetheless purposeful and further improved localization, possibly by combining two strategies. First, search was most effective when the initial target error was large. At the beginning of search, localization error diminished until it reached the overall remaining error of about $1-2 \mathrm{~cm}$. Then, however, search often continued without any further error-reducing effect, indicating that participants were probing the area close to the target, but without actually gaining higher precision. In fact, when the initial reach had already placed the searching finger near the true target location, search was overall ineffective, and sometimes even resulted in a final location report that was further from the target than the initial reach. In other words, search near the target appears to have used a random movement strategy. Alternatively, search near the target may be guided by random fluctuations of tactile sensation. In any case, search likely ended after it had not led to any perceived improvement after some time had elapsed, in line with decision theories that posit that urgency signals are instrumental in forming sensory decisions (Cisek, Puskas, \& El-Murr, 2009).

In the visual domain, it has been suggested that spatial representations are sparse for the purpose of reducing memory load and energy consumption but are effortlessly updated by sensory input generated through saccades (O'Regan \& Noë, 2001; Tatler \&
Land, 2011)-effectively relying on the "world as an outside memory” (O’Regan \& Noë, 2001; O’Regan, 1994). In this view, the sparsity of representations is not accompanied by any major functional disadvantages as they can efficiently be remedied through purposeful behavior. Both in the laboratory (Ohl et al., 2011; Srimal \& Curtis, 2010) and in naturalistic environments (Land et al., 1999), initial saccades regularly miss peripheral targets, evoking corrective saccades upon detection of the remaining error. By analogy, the present results may reflect a purposeful strategy to maintain globally sparse body representations that, when required, can be locally enhanced online by means of active search. Such a strategy appears both efficient in terms of computational costs and biologically plausible.

\section{Improvement Through Search Only Partially Depends on Tactile Input}

It is noteworthy that the unique improvement of tactile search over visually guided reaching toward the tactile target location was numerically small, and the effect barely reached significance. In fact, the availability of vision practically eliminated any tactile search activity, irrespective of initial localization error: in most trials, participants did not perform any search after touching down their finger, even though they were allowed and even encouraged to do so.

Accordingly, the tactile target must have been transformed from a skin-based, 2D spatial code into a visual or supramodal 3D spatial code that is available to the visual system. This process is commonly termed tactile remapping (Heed \& Azañón, 2014); it is thought to involve the integration of skin location with body posture to derive tactile location in space.

The spatial location estimate of tactile events appears to have a spatial resolution that is only slightly less precise than the location in $2 \mathrm{D}$ skin space. This finding has two implications. First, it suggests that the spatial code used for supramodal processing is almost as precise as that within the tactile modality alone. Second, the much higher localization error observed during search without vision must, to a large part, be due to the inability to direct the searching limb to the tactually stimulated target limb relying only on proprioception. Our results suggest that any form of online feedback that yields information about the body in space is potentially suitable to refine localization, either during the phase in which the reaching effector is transported toward the target area (as seen during visual reaching), or by actively creating tactile feedback from the target region's skin.

\section{Interruption of Perception-Action Loops by the Barrier}

Localization on a barrier that is mounted directly above the limb - that is, very close to the tactile target area-is a standard method of open-loop localization (Longo et al., 2015; J. Medina \& Duckett, 2017; Mueller \& Fiehler, 2014).

Our experiments suggest that this method obstructs tactile localization performance and results in largely inflated estimates of constant and variable localization error compared to directly pointing on the skin. Bias was strong in the lateral direction, toward the starting point of the reaching movement. This observation suggests that the barrier prematurely interrupted the hand's flight on its way to the true target location. 
Furthermore, initial localization was also more variable on the barrier, which might indicate that participants would have made final corrections in a late phase of their trajectories beyond the barrier. In line with this notion, many participants reported that the indicated position did not match well with their actual movement target. We emphasize that the barrier was attached as closely to the arm as possible without touching, and that, therefore, neither the subjective participant reports nor the large errors can be explained merely by recurring to the distance between the pointing finger and the target arm.

The purpose of using the barrier conditions was to prevent tactile while allowing for proprioceptive feedback. The presence of similar goal-directed corrective responses as observed on the skin would suggest that correction, even when searching on the skin, could also be based on proprioception rather than tactile input from the target area. In Experiment 2, localization did not improve on the barrier on average. This result suggests that tactile feedback on the searching finger, originating here from touching the barrier, does not improve localization. Accordingly, the improvement observed by tactile search on the arm can be attributed to tactile feedback specifically of the target region, supporting the idea that the mechanism underlying the search benefit is the comparison of remembered and online tactile input.

Nevertheless, some improvement was evident also during search on the barrier, namely when initial localization error was large. Moreover, improvement on the barrier was also evident in the overall average across trials in Experiment 3 and 4. These results may indicate that tactile input to the searching finger did aid localization after all. Several reasons make this interpretation unlikely. First, errors on the barrier were generally considerably larger than on the skin. Second, both initial and final localization error was several times larger than on the skin, especially in Experiments 3 and 4 . This makes it likely that the discrepancy between target and searching hand was sensed proprioceptively; recall that participants even spontaneously reported such concerns. Third, when we allowed vision in Experiment 4, the localization error dropped by a large amount, suggesting that vision allowed correction of the large, proprioceptive error. Finally and most importantly, the improvement from initial to final error was larger when participants searched on the skin than when they searched on the barrier. If tactile information from the searching finger were instrumental to search success, no such discrepancy should be present.

These considerations lead us to suggest that the mechanism of localization improvement differs between skin and barrier: on the skin, improvement is driven by tactile information from the target region even in close vicinity of the target; in contrast, on the barrier, improvement is driven by proprioception and, thus, can only be effective at a coarse spatial resolution, so that only large localization errors are detected and corrected to a small degree.

\section{Similar Bias in Open-Loop and Closed-Loop Localization}

Finally, the finding that the closed-loop method used here is associated with more accurate localization than open-loop methods does not automatically imply that results obtained with open-loop methods are invalid. We contend that pointing methods that ask participants to indicate tactile locations on a barrier, in the air, or on a drawing are very likely to overestimate error and, accordingly, underestimate absolute localization ability.

However, other behaviors may be observable using either of the localization methods. For instance, the interrupted reaching when using a barrier may reveal qualitatively similar bias if the reaching movements are similar from trial to trial, which is usually the case. Similarly, it may be possible to reduce barrier effects as those demonstrated here by instructing pointing movements that are directed downward onto a barrier placed parallel to the table, as one might expect that the hand could then be located over the to-be-localized skin location during flight and the finger would, thus, end up directly above the skin location that the movement is targeted at. However, under which circumstances the methods are equivalent and when they differ should be further investigated by future research.

For distal-direction biases known to exist for open-loop localizations near the wrist, for example, our results matched those of earlier reports (Longo et al., 2015; Mancini, Longo, Iannetti, et al., 2011). However, other biases and representational distortions may be sensitive to closed-loop tactile feedback.

Finally, indicating a tactile location with visual guidance was surprisingly accurate in comparison to on-skin search. Experimental paradigms often prevent vision with the aim to test "pure" tactile ability. Yet, our results suggest that errors observed when localizing without vision may be indicative of proprioceptive-motor, rather than tactile-representational, accuracy. Thus, maybe counterintuitively, the present results suggest that if an experiment aims to assess precise localization ability without allowing touch to the target region, participants should be allowed to view their reaching and target arms. Moreover, vision also led participants, against instructions, to refrain from searching, which would have improved their localization performance. Thus, the highest accuracy of tactile localization will be achieved by preventing participants to see their reaching and target arms, but to allow them to search for the tactile target on the skin.

\section{Conclusion}

Tactile localization is a closed-loop, active process, in which online sensory input reduces localization error. We showed that tactile input from the target region on the skin can markedly improve localization performance. Yet, vision can update perception of the spatial relationships between the reaching and target limbs, leading to localization that is almost as precise as that achieved through (blind) tactile search. These results imply that movements to tactile events are guided by a body representation that is almost as precise as what can be achieved through online search when relying on touch alone. It has been suggested that active perception can offload requirements for high spatial resolution from cognitive representations to perceptual strategies that are evoked online when high resolution is needed. Our everyday behavior of searching locations on the skin seems to support this idea. However, our experiments suggest that the benefit of active touch is small, and that the reason for large corrections when we cannot see the touched region may not primarily be strategic savings in our brain's maps but rather the low spatial resolution of our proprioceptive sense. 


\section{References}

Ahissar, E., \& Arieli, A. (2001). Figuring space by time. Neuron, 32, 185-201. http://dx.doi.org/10.1016/S0896-6273(01)00466-4

Alais, D., \& Burr, D. (2004). The ventriloquist effect results from nearoptimal bimodal integration. Current Biology, 14, 257-262. http://dx.doi .org/10.1016/j.cub.2004.01.029

Badde, S., \& Heed, T. (2016). Towards explaining spatial touch perception: Weighted integration of multiple location codes. Cognitive Neuropsychology, 33(1-2), 26-47. http://dx.doi.org/10.1080/02643294.2016 .1168791

Bates, D., Mächler, M., Bolker, B., \& Walker, S. (2014). Fitting linear mixed-effects models using lme4. ArXiv Preprint ArXiv:1406.5823. Retrieved from http://arxiv.org/abs/1406.5823

Calzolari, E., Azañón, E., Danvers, M., Vallar, G., \& Longo, M. R. (2017). Adaptation aftereffects reveal that tactile distance is a basic somatosensory feature. Proceedings of the National Academy of Sciences of the United States of America, 114, 4555-4560. http://dx.doi.org/10.1073/ pnas. 1614979114

Cisek, P., Puskas, G. A., \& El-Murr, S. (2009). Decisions in changing conditions: The urgency-gating model. The Journal of Neuroscience, 29, 11560-11571. http://dx.doi.org/10.1523/JNEUROSCI.1844-09.2009

Culver, C. M. (1970). Errors in tactile localization. The American Journal of Psychology, 83, 420-427. http://dx.doi.org/10.2307/1420418

Desmurget, M., Epstein, C. M., Turner, R. S., Prablanc, C., Alexander, G. E., \& Grafton, S. T. (1999). Role of the posterior parietal cortex in updating reaching movements to a visual target. Nature Neuroscience, 2 , 563-567. http://dx.doi.org/10.1038/9219

Elithorn, A., Piercy, M. F., \& Crosskey, M. A. (1953). Tactile localization. The Quarterly Journal of Experimental Psychology, 5, 171-182. http:// dx.doi.org/10.1080/17470215308416640

Ernst, M. O., \& Banks, M. S. (2002). Humans integrate visual and haptic information in a statistically optimal fashion. Nature, 415, 429-433. http://dx.doi.org/10.1038/415429a

Friston, K. (2010). The free-energy principle: A unified brain theory? Nature Reviews Neuroscience, 11, 127-138. http://dx.doi.org/10.1038/ nrn2787

Fuentes, C. T., \& Bastian, A. J. (2010). Where is your arm? Variations in proprioception across space and tasks. Journal of Neurophysiology, 103, 164-171. http://dx.doi.org/10.1152/jn.00494.2009

Gallace, A., \& Spence, C. (2009). The cognitive and neural correlates of tactile memory. Psychological Bulletin, 135, 380-406. http://dx.doi.org/ 10.1037/a0015325

Geldard, F. A. (1985). The mutability of time and space on the skin. The Journal of the Acoustical Society of America, 77, 233-237. http://dx.doi .org/10.1121/1.392264

Haggard, P., Taylor-Clarke, M., \& Kennett, S. (2003). Tactile perception, cortical representation and the bodily self. Current Biology, 13(5), R170-R173. http://dx.doi.org/10.1016/S0960-9822(03)00115-5

Harris, C. M., \& Wolpert, D. M. (1998). Signal-dependent noise determines motor planning. Nature, 394, 780-784. http://dx.doi.org/10.1038/ 29528

Head, H. (1920). Studies in neurology. Retrieved from http://archive.org/ details/studiesinneurolo01headiala

Heed, T., \& Azañón, E. (2014). Using time to investigate space: A review of tactile temporal order judgments as a window onto spatial processing in touch. Frontiers in Psychology, 5, 76. http://dx.doi.org/10.3389/fpsyg .2014 .00076

Heed, T., Buchholz, V. N., Engel, A. K., \& Röder, B. (2015). Tactile remapping: From coordinate transformation to integration in sensorimotor processing. Trends in Cognitive Sciences, 19, 251-258. http://dx.doi .org/10.1016/j.tics.2015.03.001

Kammers, M. P. M., de Vignemont, F., Verhagen, L., \& Dijkerman, H. C. (2009). The rubber hand illusion in action. Neuropsychologia, 47, 204211. http://dx.doi.org/10.1016/j.neuropsychologia.2008.07.028
Kieslich, P. J., Henninger, F., Wulff, D. U., Haslbeck, J. M. B., \& Schulte-Mecklenbeck, M. (2019). Mouse-tracking: A practical guide to implementation and analysis. In M. Schulte-Mecklenbeck, A. Kühberger, \& J. G. Johnson (Eds.), A handbook of process tracing methods (pp. 111-130). New York, NY: Routledge.

Króliczak, G., Heard, P., Goodale, M. A., \& Gregory, R. L. (2006). Dissociation of perception and action unmasked by the hollow-face illusion. Brain Research, 1080, 9-16. http://dx.doi.org/10.1016/j .brainres.2005.01.107

Kuznetsova, A., Brockhoff, P. B., \& Christensen, R. H. B. (2014). lmer Test: Tests for random and fixed effects for linear mixed effect models (lmer objects of lme4 package). Retrieved from http://CRAN.R-project .org/package $=$ lmerTest

Land, M., Mennie, N., \& Rusted, J. (1999). The roles of vision and eye movements in the control of activities of daily living. Perception, 28 1311-1328. http://dx.doi.org/10.1068/p2935

Lederman, S. J., \& Klatzky, R. L. (2009). Haptic perception: A tutorial. Attention, Perception, \& Psychophysics, 71, 1439-1459. http://dx.doi .org/10.3758/APP.71.7.1439

Lenth, R., Singmann, H., Love, J., Buerkner, P., \& Herve, M. (2019). emmeans: Estimated Marginal Means, aka Least-Squares Means (Version 1.4.3.01). Retrieved from https://CRAN.R-project.org/ package $=$ emmeans

Longo, M. R., \& Haggard, P. (2010). An implicit body representation underlying human position sense. Proceedings of the National Academy of Sciences of the United States of America, 107, 11727-11732. http:// dx.doi.org/10.1073/pnas.1003483107

Longo, M. R., \& Haggard, P. (2011). Weber's illusion and body shape: Anisotropy of tactile size perception on the hand. Journal of Experimental Psychology: Human Perception and Performance, 37, 720-726. http://dx.doi.org/10.1037/a0021921

Longo, M. R., Mancini, F., \& Haggard, P. (2015). Implicit body representations and tactile spatial remapping. Acta Psychologica, 160, 77-87. http://dx.doi.org/10.1016/j.actpsy.2015.07.002

Maij, F., Medendorp, W. P., \& Heed, T. (2019). External location of touch is constructed post-hoc based on limb choice. bioRxiv. Advance online publication. http://dx.doi.org/10.1101/549832

Maij, F., Wing, A. M., \& Medendorp, W. P. (2013). Spatiotemporal integration for tactile localization during arm movements: A probabilistic approach. Journal of Neurophysiology, 110, 2661-2669. http://dx.doi .org/10.1152/jn.00971.2012

Mancini, F., Longo, M. R., Iannetti, G. D., \& Haggard, P. (2011). A supramodal representation of the body surface. Neuropsychologia, 49, 1194-1201. http://dx.doi.org/10.1016/j.neuropsychologia.2010.12.040

Mancini, F., Longo, M. R., Kammers, M. P. M., \& Haggard, P. (2011). Visual distortion of body size modulates pain perception. Psychological Science, 22, 325-330. http://dx.doi.org/10.1177/0956797611398496

Medina, J., \& Duckett, C. (2017). Domain-general biases in spatial localization: Evidence against a distorted body model hypothesis. Journal of Experimental Psychology: Human Perception and Performance, 43, 1430-1443. http://dx.doi.org/10.1037/xhp0000397

Medina, S., Tamè, L., \& Longo, M. R. (2018). Tactile localization biases are modulated by gaze direction. Experimental Brain Research, 236, 31-42. http://dx.doi.org/10.1007/s00221-017-5105-2

Mueller, S., \& Fiehler, K. (2014). Effector movement triggers gazedependent spatial coding of tactile and proprioceptive-tactile reach targets. Neuropsychologia, 62, 184-193. http://dx.doi.org/10.1016/j .neuropsychologia.2014.07.025

Ohl, S., Brandt, S. A., \& Kliegl, R. (2011). Secondary (micro-)saccades: The influence of primary saccade end point and target eccentricity on the process of postsaccadic fixation. Vision Research, 51, 2340-2347. http://dx.doi.org/10.1016/j.visres.2011.09.005 
Oldfield, R. C. (1971). The assessment and analysis of handedness: The Edinburgh inventory. Neuropsychologia, 9, 97-113. http://dx.doi.org/10 .1016/0028-3932(71)90067-4

O'Regan, J. K. (1994). The world as an outside iconic memory-no strong internal metric means no problem of visual stability. Behavioral and Brain Sciences, 17, 270-271. http://dx.doi.org/10.1017/ S0140525X00034488

O'Regan, J. K., \& Noë, A. (2001). A sensorimotor account of vision and visual consciousness. Behavioral and Brain Sciences, 24, 939-973. http://dx.doi.org/10.1017/S0140525X01000115

Press, C., Taylor-Clarke, M., Kennett, S., \& Haggard, P. (2004). Visual enhancement of touch in spatial body representation. Experimental Brain Research, 154, 238-245. http://dx.doi.org/10.1007/s00221-0031651-x

$\mathrm{R}$ Core Team. (2014). $R$ : A language and environment for statistical computing. Retrieved from http://www.R-project.org/

Risi, N., Shah, V., Mrotek, L. A., Casadio, M., \& Scheidt, R. A. (2019). Supplemental vibrotactile feedback of real-time limb position enhances precision of goal-directed reaching. Journal of Neurophysiology, 122, 22-38. http://dx.doi.org/10.1152/jn.00337.2018

Schlager, S. (2017). Morpho and Rvcg—-shape analysis. In G. Zheng, S. Li, \& G. Szekely (Eds.), Statistical shape and deformation analysis (pp. 217-256). London, UK: Academic Press. http://dx.doi.org/10.1016/ B978-0-12-810493-4.00011-0

Scott, S. H. (2004). Optimal feedback control and the neural basis of volitional motor control. Nature Reviews Neuroscience, 5, 532-545. http://dx.doi.org/10.1038/nrn1427

Smeets, J. B., van den Dobbelsteen, J. J., de Grave, D. D., van Beers, R. J., \& Brenner, E. (2006). Sensory integration does not lead to sensory calibration. Proceedings of the National Academy of Sciences of the United States of America, 103, 18781-18786. http://dx.doi.org/10.1073/ pnas.0607687103

Spence, C., Nicholls, M. E., Gillespie, N., \& Driver, J. (1998). Cross-modal links in exogenous covert spatial orienting between touch, audition, and vision. Perception \& Psychophysics, 60, 544-557. http://dx.doi.org/10 $.3758 / \mathrm{BF} 03206045$

Srimal, R., \& Curtis, C. E. (2010). Secondary adaptation of memoryguided saccades. Experimental Brain Research, 206, 35-46. http://dx .doi.org/10.1007/s00221-010-2394-0
Steenbergen, P., Buitenweg, J. R., Trojan, J., \& Veltink, P. H. (2014) Tactile localization depends on stimulus intensity. Experimental Brain Research, 232, 597-607. http://dx.doi.org/10.1007/s00221-013-3768-x

Tamè, L., Azañón, E., \& Longo, M. R. (2019). A conceptual model of tactile processing across body features of size, shape, side, and spatial location. Frontiers in Psychology, 10, 291. http://dx.doi.org/10.3389/ fpsyg.2019.00291

Tatler, B. W., \& Land, M. F. (2011). Vision and the representation of the surroundings in spatial memory. Philosophical Transactions of the Royal Society of London Series B, Biological Sciences, 366, 596-610. http://dx.doi.org/10.1098/rstb.2010.0188

Taylor-Clark, M., Kennett, S., \& Haggard, P. (2002). Vision modulates somatosensory cortical processing. Current Biology, 12, 233-236. http:// dx.doi.org/10.1016/S0960-9822(01)00681-9

Trojan, J., Stolle, A. M., Carl, A. M., Kleinböhl, D., Tan, H. Z., \& Hölzl, R. (2010). Spatiotemporal integration in somatosensory perception: Effects of sensory saltation on pointing at perceived positions on the body surface. Frontiers in Psychology, 1, 206. Advance online publication. http://dx.doi.org/10.3389/fpsyg.2010.00206

Trommershäuser, J., Maloney, L. T., \& Landy, M. S. (2008). Decision making, movement planning and statistical decision theory. Trends in Cognitive Sciences, 12, 291-297. http://dx.doi.org/10.1016/j.tics.2008 .04 .010

Weinstein, S. (1968). Intensive and extensive aspects of tactile sensitivity as a function of body part, sex, and laterality. In D. R. Kenshalo (Ed.) The skin senses: Proceedings of the first International Symposium on the Skin Senses (pp. 195-218). Springfield, IL: Charles C Thomas Publishing.

Wulff, D. U., Haslbeck, J. M. B., Kieslich, P. J., Henninger, F., \& Schulte-Mecklenbeck, M. (2019). Mouse-tracking: Detecting types in movement trajectories. In M. Schulte-Mecklenbeck, A. Kühberger, \& J. G. Johnson (Eds.), A handbook of process tracing methods (pp. 131-145). New York, NY: Routledge.

Received June 5, 2019

Revision received January 3, 2020

Accepted February 18, 2020

\section{E-Mail Notification of Your Latest Issue Online!}

Would you like to know when the next issue of your favorite APA journal will be available online? This service is now available to you. Sign up at https://my.apa.org/portal/alerts/ and you will be notified by e-mail when issues of interest to you become available! 